\title{
Distinct projections from the infralimbic cortex exert opposing effects in modulating anxiety and fear
}

\author{
Yi-Hua Chen, Jian-Lin Wu, Neng-Yuan Hu, Jia-Pai Zhuang, Wei-Peng Li, Sheng-Rong Zhang, Xiao-Wen Li, Jian-Ming Yang, \\ and Tian-Ming Gao \\ State Key Laboratory of Organ Failure Research, Key Laboratory of Mental Health of the Ministry of Education, Guangdong-Hong Kong-Macao Greater Bay Area Center for Brain Science and Brain-Inspired \\ Intelligence, Guangdong Province Key Laboratory of Psychiatric Disorders, Department of Neurobiology, School of Basic Medical Sciences, Southern Medical University, Guangzhou, China.
}

\begin{abstract}
Anxiety-related disorders can be treated by cognitive therapies and transcranial magnetic stimulation, which involve the medial prefrontal cortex (MPFC). Subregions of the MPFC have been implicated in mediating different and even opposite roles in anxiety-related behaviors. However, precise causal targets of these top-down connections among diverse possibilities have not been established. Here, we show that the lateral septum (LS) and the central nucleus of the amygdala (CeA) represent 2 direct targets of the infralimbic cortex (IL), a subregion of the mPFC that modulates anxiety and fear. Two projections were unexpectedly found to exert opposite effects on the anxious state and learned freezing: the IL-LS projection promoted anxiety-related behaviors and fear-related freezing, whereas the IL-CeA projection exerted anxiolytic and fear-releasing effects for the same features. Furthermore, selective inhibition of corresponding circuit elements showed opposing behavioral effects compared with excitation. Notably, the IL-CeA projection implemented top-down control of the stress-induced high-anxiety state. These results suggest that distinct IL outputs exert opposite effects in modulating anxiety and fear and that modulating the excitability of these projections with distinct strategies may be beneficial for the treatment of anxiety disorders.
\end{abstract}

\section{Introduction}

Anxiety disorders represent the most common psychiatric diseases, with a combined lifetime prevalence of over $28 \%(1,2)$. Although the available pharmacological treatments are inadequate $(3)$, cognitive therapies $(4,5)$ and transcranial magnetic stimulation (6-8) have shown effective therapeutic effects. There has therefore been interest in understanding how cognition regulates affect, thus providing pathways for intervention. Increasing evidence has suggested that the medial prefrontal cortex (mPFC), i.e., the cognitive control region, could be involved in anxiety and fear regulation in both humans and rodents (9-12). The mPFC exerts concerted top-down control over subcortical regions to tune the expression of fear and anxiety (13-18). Previous studies have suggested that subregions of the $\mathrm{MPFC}$ have different and even opposite roles in modulating fear (19-22). However, precise causal targets of these top-down connections among diverse possibilities have not been established.

The infralimbic cortex (IL) is a major component of the MPFC. Lesions of the rodent IL have been shown to decrease anxiety-like behaviors in the elevated plus maze, shock-probe burying, and Vogel conflict tests $(23,24)$. Our recent experiments have demonstrated that pharmacologically enhanced excitability in the IL produces anxiety-like behaviors (25). Electrical stimulation of the IL decreases

Conflict of interest: The authors have declared that no conflict of interest exists. Copyright: () 2021, American Society for Clinical Investigation.

Submitted: November 2, 2020; Accepted: June 3, 2021; Published: July 15, 2021

Reference information: J Clin Invest. 2021;131(14):e145692.

https://doi.org/10.1172/JCl145692. conditioned fear responses (21) and facilitates fear extinction (26). Consistently, pharmacological inactivation of the IL impairs fear extinction and extinction retrieval (27). The function of the IL in regulating the extinction of fear memory seems to be contradictory to that in regulating anxiety-like behaviors, as mentioned above. The difference may be because the IL contains different cell types that project to different targets $(28,29)$. Lesions, extracellular electrical manipulations, and pharmacological treatments lack specificity for cell types and temporal precision (30). One report showed that optogenetic activation of IL neurons modulates extinction (31). However, no studies have thus far addressed the behavioral variety in top-down projections in real time.

Considerable effort has been made to map the fear and anxiety neural circuits, most recently using optogenetic tools. The amygdala, extended amygdala, and septo-hippocampal axis play important roles $(32,33)$. Optogenetic activation of $\mathrm{Crfr}^{2}{ }^{+}$neurons in the lateral septum (LS) exerts an anxiogenic effect (34). The amygdala microcircuit - basolateral amygdala (BLA) to central nucleus of the amygdala $(\mathrm{CeA})$ - mediates reversible and bidirectional control of anxiety (35). Furthermore, an inhibitory microcircuit exists within the CeA and consists of the lateral and medial subdivisions that mediate the acquisition and expression of conditioned fear, respectively, in mice (36-38). Although this amygdala microcircuit is well positioned to be influenced by top-down cortical control from regions important for processing fear and anxiety, including the IL, which provides innervations to the BLA and CeA $(28,29)$, it has received little attention.

In this study, we sought to examine the effects of acute manipulation of the activity of the IL and its distal projections 
on anxiety-related behavior in freely moving mice. We identified a functional role for the IL-LS and IL-CeA pathways in opposite directions in modulating anxiety and fear and elucidated a potential role for the IL-CeA circuit in the treatment of stress-induced anxiety.

\section{Results}

Optogenetic activation of the IL increases anxiety-like behavior. To locally express opsin in the IL, we used adeno-associated viral vector serotype 2 (AAV2), which has shown a relatively restricted expression pattern in the CNS (39-41). AAV2 vectors encoding ChR2, a nonspecific cation channel derived from the green algae Chlamydomonas reinhardtii, fused in-frame to enhance yellow fluorescent protein (ChR2-eYFP) under the control of the calcium/ calmodulin-dependent protein kinase II $\alpha$ (CaMKII $\alpha)$ promoter were used to selectively activate excitatory glutamatergic neurons. Stereotactic delivery of the ChR2-eYFP vectors resulted in IL-specific expression (Figure 1A and Supplemental Figure 1A; supplemental material available online with this article; https:// doi.org/10.1172/JCI145692DS1). Whole-cell recordings from brain slices demonstrated that ChR2-eYFP ${ }^{+}$neurons in the IL were reliably activated by blue light $(473 \mathrm{~nm})$ pulses and faithfully fired action potentials at a frequency of up to $40 \mathrm{~Hz}$ (Figure 1B).

To test whether the IL is essential in the regulation of anxiety, we first probed freely moving mice under optogenetic activation of IL glutamatergic neurons in 2 well-validated anxiety assays (42), the elevated plus maze (EPM) test and the open field test (OFT). The experimental paradigm is shown in Figure 1C. IL neurons were transduced with ChR2 in the experimental animals (referred to hereafter as ChR2 mice), whereas the control animals, matched for age, incubation duration, and illumination parameters, received the same viral vector carrying the fluorophore alone (referred to hereafter as eYFP mice). To activate ChR2-expressing IL neurons, optical fibers were unilaterally implanted above the IL in the ipsilateral hemisphere for the delivery of blue light (Supplemental Figure 1, A and B). To allow within-subjects and within-sessions comparisons in addition to group comparisons, we tested mice in a single 9-minute session of both the EPM test and the OFT in three 3-minute epochs, beginning with a light-off (OFF) baseline epoch, followed by a light-on (ON) illumination epoch using a continuous train of blue light $(473 \mathrm{~nm})$ pulses at 20 $\mathrm{Hz}$ with a 5-millisecond pulse duration (13), and then alternating back to a second OFF epoch. A representative EPM animal track from a mouse in the ChR2 group is shown across epochs (Figure 1D). Relative to the eYFP control mice, mice in the ChR2 group showed significantly reduced open-arm exploration (Figure 1E) as well as a lower probability of open-arm entry (Figure 1F), reflecting increased anxiety-related behaviors during the ON epoch and beyond the ON epoch.

We further evaluated the effects of IL activation on anxietyrelated behaviors and locomotor activity in the OFT. Using the same illumination parameters as in the EPM test, a representative animal track from a mouse in the ChR2 group is shown across epochs (Figure 1G). Consistent with our EPM results, relative to the eYFP control mice, the ChR2 mice showed a decrease in center exploration, reflecting anxiogenic effects upon illumination in the ON epoch, and the effect outlasted the laser stimulation (Figure
1H). Importantly, in the ChR2 group, we did not detect an effect of light on locomotion, as measured by distance traveled (Figure 1I).

The dorsal peduncular cortex (DP), the other component of the ventral $\mathrm{mPFC}$ (vmPFC), is located next to the IL. A previous study reported that exciting the entire vmPFC (including both the IL and DP) had no effect on anxiety-like behaviors (13). We next sought to identify the effects of direct excitation of the DP on anxiety-like behaviors. We injected viruses locally into the DP (Supplemental Figure 2, A and G). Using the same protocol as shown in Figure 1 (Supplemental Figure 2B), we found that DP activation increased open-arm exploration (Supplemental Figure 2C), as well as the probability of open-arm entry (Supplemental Figure 2D) on the EPM test and central exploration in the OFT (Supplemental Figure 2E), without affecting locomotion (Supplemental Figure 2F), suggesting an anxiolytic effect. Since IL activation is anxiogenic (Figure 1, D-I), it may counterbalance the anxiolytic effect of DP excitation when the whole vmPFC is excited.

These results suggested that activating IL glutamatergic neuronal activity robustly induced an anxiogenic effect. Next, we explored whether inhibition of the IL could control anxiety in a bidirectional manner.

Optogenetic inhibition of the IL reduces anxiety-like behavior. To test whether inhibition of the IL was sufficient to cause a decrease in anxiety-related behaviors, we induced expression of the eNpHR3.0-eYFP fusion protein (eNpHR-eYFP) in IL excitatory glutamatergic neurons in experimental animals (Figure 1J and Supplemental Figure 1C) as well as expression of eYFP alone in control animals and bilaterally implanted optical fibers above the IL (Figure 1L). Whole-cell recordings from brain slices demonstrated that the eNpHR-eYFP ${ }^{+}$neurons were hyperpolarized by continuous yellow light $(594 \mathrm{~nm}$ ) illumination (Figure $1 \mathrm{~K})$. We conducted the same 9-minute session with alternating OFF-ONOFF epochs as in the previous experiments, but used constant illumination with 594-nm light in the illumination epoch. Relative to the eYFP controls, we observed that the mice in the eNpHR group (referred to hereafter as eNpHR mice) showed greater open-arm exploration (Figure 1M) during the $\mathrm{ON}$ epoch, as well as a higher probability of open-arm entry (Figure $1 \mathrm{~N}$ ). We also subjected mice to the OFT. Relative to the eYFP control mice, the eNpHR mice showed an increase in center exploration, reflecting anxiolytic effects upon illumination in the ON epoch (Figure 1O), consistent with our EPM results. Again, we simultaneously assayed locomotor activity across epochs and did not detect any changes, as measured by distance traveled (Figure 1P). These data demonstrated that inhibition of IL glutamatergic neuronal activity robustly and reversibly induced an anxiolytic effect on the order of seconds.

Distinct IL outputs modulate anxiety states in an antagonistic manner. We next sought to identify the neural circuit targets underlying IL top-down modulation of anxiety-related behavior. For systematic mapping of brain regions that are directly innervated by the IL, we stereotaxically injected AAV2-ChR2-eYFP into the IL (Supplemental Figure 3A). In addition to well-characterized outputs from the IL, such as those to the thalamus (Supplemental Figure 3C) and the dorsal raphe nucleus (Supplemental Figure $3 \mathrm{D}$ ), we also detected eYFP-expressing neurons in the LS (Figure $2 \mathrm{~A}$ and Supplemental Figure 3E), CeA (Figure 2B and Supplemental Figure 3F), BLA (Figure $2 \mathrm{~B}$ and Supplemental Figure 3F), and 
A



B



$20 \mathrm{~Hz}$

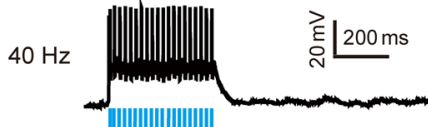

C

Virus injection Fiber implantation EPM + light $\quad$ OFT + light



$\mathbf{E}$



J

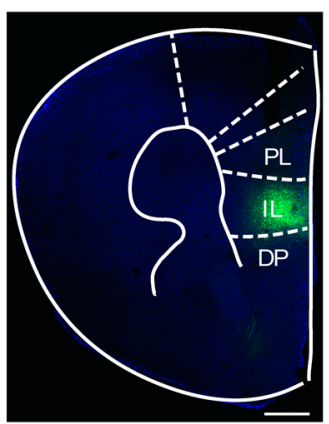

M



$\mathbf{F}$

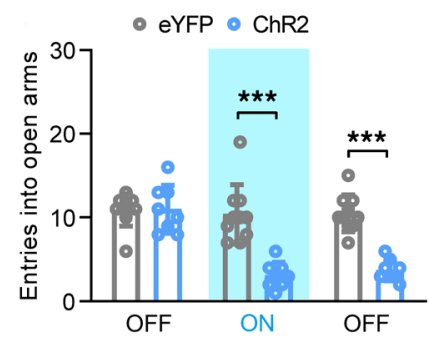

H



I



$\mathbf{L}$

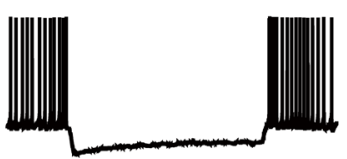

产2s
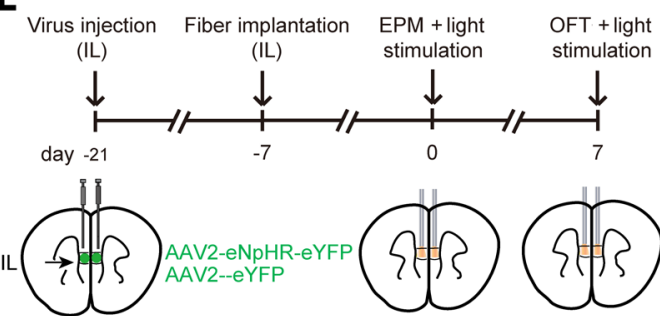

$\mathbf{N}$

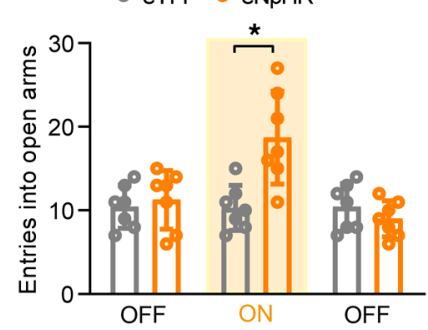

o

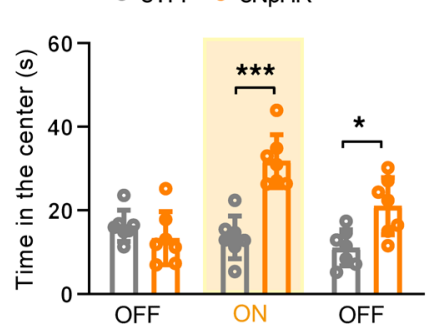

$\mathbf{P}$

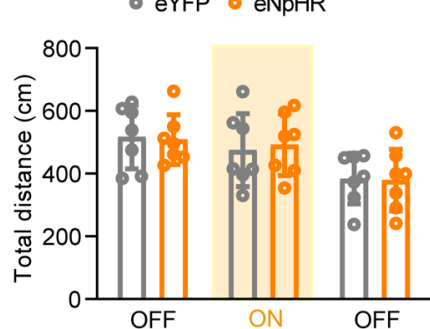


Figure 1. The IL is implicated in anxiety-like behavior. (A) Expression of ChR2 in the IL. Scale bar: $500 \mu \mathrm{m}$. M2, secondary motor cortex; Cg1, cingulate cortex, area 1; PL, prelimbic cortex. (B) Brief blue light pulses at $5 \mathrm{~Hz}, 20 \mathrm{~Hz}$, and $40 \mathrm{~Hz}$ precisely activated ChR2 cells. (C) Schematic protocol for investigating the behavioral impact of optogenetic activation of IL somata. (D) Representative animal track across epochs in the EPM for a ChR2 mouse. Mice were tested in 3-minute epochs across a 9-minute session. (E) ChR2 mice $(n=10)$ spent less time in the open arms than did eYFP mice $(n=8)$ during photostimulation $\left[F_{\text {interaction }}(2,32)=3.959, P=\right.$ $0.0291]$. (F) ChR2 mice showed a lower probability of entering the open arms than did eYFP mice during photostimulation $\left[F_{\text {interaction }}(2,32)=10.88\right.$, $P=0.0002]$. (G) Representative animal track across epochs in the OFT for a ChR2 mouse. (H) During the illumination epoch, ChR2 mice spent less time exploring the center of the open field than did eYFP mice $\left[F_{\text {interaction }}(2,32)=\right.$ 21.12, $P<0.0001]$. (I) Photostimulation did not alter the distance traveled for mice in either group. (J) Expression of eNpHR in the IL. Scale bar: 500 $\mu \mathrm{m}$. (K) Yellow light illumination of eNpHR cells in the IL blocked evoked spiking. (L) Schematic protocol for investigating the behavioral impact of optogenetic inhibition of IL somata. (M) Same as in $\mathbf{E}$ but for eNpHR mice $\left[n=7\right.$ per group, $\left.F_{\text {interaction }}(2,24)=4.452, P=0.0227\right]$. (N) Same as in F but for eNpHR mice $\left[F_{\text {interaction }}(2,24)=7.726, P=0.0026\right]$. (0) Same as in $\mathbf{H}$ but for eNpHR mice $\left[F_{\text {interaction }}(2,24)=11.76, P=0.0003\right]$. (P) Same as in I but for eNpHR mice. ${ }^{*} P<0.05,{ }^{* *} P<0.01$, and ${ }^{* *} P<0.001$, by 2 -way, repeated-measures ANOVA with Bonferroni's post hoc analysis (E-I and M-P). Data are presented as the mean \pm SEM. See Supplemental Table 1 for statistical details.

bed nucleus of the stria terminalis (BNST) (Supplemental Figure 3B). These results were particularly interesting, because although previous studies have suggested a potentially important role for the LS, CeA, BLA, and BNST in anxiety-related behaviors $(34,35$, $43)$, whether these regions are under top-down cortical control from the IL remains unknown.

To test whether activation of IL axons in the LS, CeA, BLA, and BNST was sufficient to modulate anxiety-related behaviors, we expressed ChR2-eYFP in the IL in experimental animals and eYFP alone in the IL in control animals and unilaterally implanted an optical fiber over one of the above-mentioned regions in the ipsilateral hemisphere prior to anxiety testing (Figure 2, C, D, and I, and Supplemental Figure 4, A and B). Relative to the eYFP controls, the animals in the ChR2 IL-LS group showed decreased open-arm exploration (Figure 2E) and a lower probability of openarm entry (Figure 2F) on the EPM test, as well as a decrease in center exploration time (Figure $2 \mathrm{G}$ ) in the OFT during the $\mathrm{ON}$ epoch, reflecting anxiogenic effects. In contrast, relative to the eYFP controls, the animals in the ChR2 IL-CeA group showed increased open-arm exploration (Figure 2J), a higher probability of open-arm entry (Figure 2K), and an increase in center exploration time (Figure 2L) in the OFT during the ON epoch, reflecting reductions in anxiety-related behaviors. Importantly, we did not detect an effect of light in any group on locomotion, as measured by the distance traveled (Figures 2, $\mathrm{H}$ and $\mathrm{M}$ ). Although there was denser innervation in the BLA than in the CeA (Figure 2B and Supplemental Figures 3F, 4B, and 8C), optical stimulation did not affect anxiety-related behaviors in the ChR2 IL-BLA animals in either assay (Supplemental Figure 5). In addition, optical stimulation did not affect anxiety-related behaviors in the ChR2 IL-BNST animals (Supplemental Figure 5).

To determine whether the robust changes in anxiety-related behaviors that we observed were indeed mediated by glutama- tergic inputs from the IL into the LS or CeA, we performed an additional series of experiments. The experimental paradigm is shown in Figure 3A. We induced expression of ChR2 in IL projection neurons as before and implanted guide cannulas to deliver either saline or glutamate receptor antagonists to the LS or CeA 30 minutes prior to testing and illumination in the EPM or OFT (Figure 3, B and G, and Supplemental Figure 6). We compared saline with a glutamate receptor antagonist cocktail, in which the combination of the $\alpha$-amino-3-hydroxy-5-methyl-4-isoxazolepropionic acid (AMPA) and NMDA receptor antagonists NBQX and AP5, respectively, was intracranially administered into the LS or CeA. In the saline IL-LS trials, mice replicated the light-induced anxiogenic effect in both the EPM test (Figure 3, C and D) and the OFT (Figure 3, E and F). However, preapplication of glutamate receptor antagonists completely blocked light-induced changes in open-arm exploration on the EPM test (Figure 3, C and D) and the time spent in the center in the OFT (Figure 3, E and F). In parallel experiments, the mice duplicated the light-induced anxiolytic effect in both the EPM test and the OFT in the saline IL-CeA trials. Again, the light-induced changes in open-arm exploration in the EPM test (Figure 3, H and I) and the time spent in the center in the OFT (Figure 3, J and K) were prevented by antagonist treatment.

Taken together, these data suggested that IL axon terminals synapsing locally in the LS and the CeA can modulate anxietyrelated behaviors in opposite directions.

Monosynaptic and functional IL inputs into the LS or CeA. To determine whether the IL directly innervates the LS and CeA, we applied 3 strategies. First, we injected red fluorescent retrobeads into the LS or CeA of C57 mice and found LS-projecting (Figure 4, A and B) and CeA-projecting (Figure 4, D and E) IL neurons, respectively, 2 weeks later. Second, we conducted a modified intersectional approach involving 2 viral vectors (44). In this method, Cre recombinase was delivered from retrograde canine adenovirus type 2 (CAV-Cre) (45), which transduces axon terminals and thereby defines "starter cells" by virtue of their projecting target. We injected CAV-Cre into either the LS or CeA and then injected AAV-expressing, Cre-dependent opsin ChR2 fused to mCherry into the IL (Figure 4, A and D). Four weeks later, putative starter cells in the IL were labeled, as defined by neurons that expressed mCherry, and named LS- and CeA-projecting IL neurons, respectively (Figure 4, C and F). Finally, we used ex vivo whole-cell patch-clamp recordings in brain slices containing the LS or CeA from mice expressing ChR2-eYFP in IL neurons and axon terminals. We observed that the light-evoked postsynaptic currents were completely blocked by the application of tetrodotoxin (TTX) and recovered by $4 \mathrm{AP}$, indicating that the postsynaptic currents recorded in the LS (Figure 4, G-I) or CeA (Figure 4, K-M) were elicited by direct synaptic connections from IL neurons. We also found that light-evoked excitatory postsynaptic currents (EPSCs) had a short latency (Figure 4, J and $\mathrm{N}$ ). In addition, we confirmed that these terminals released glutamate, as we found that the addition of NBQX abolished the light-induced excitation in these same cells (Figure 4, I and M). Taken together, these data suggested that LS and CeA neurons receive monosynaptic input from IL neurons. Next, we addressed the question of whether neurons projecting to the LS and CeA have a different pattern of distribution. By injecting the retrograde tracer recombinant cholera toxin subunit B (CTB) of 
A

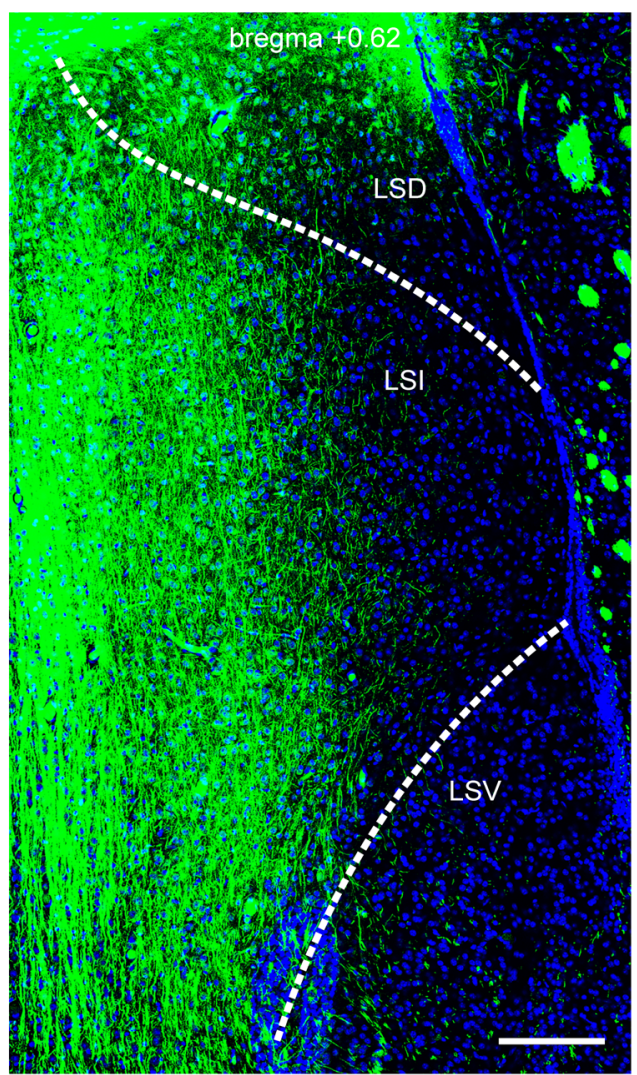

B

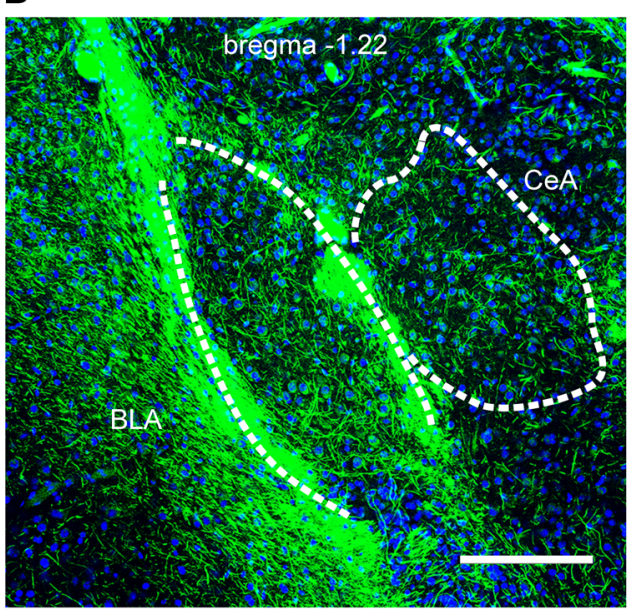

C

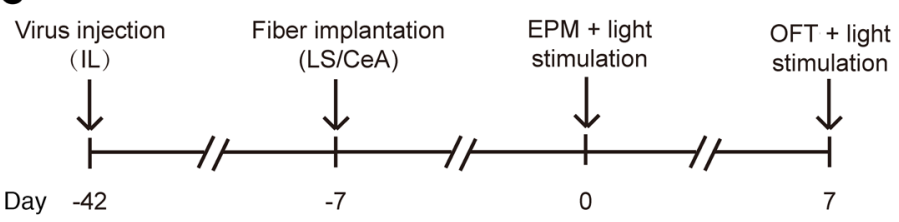

D

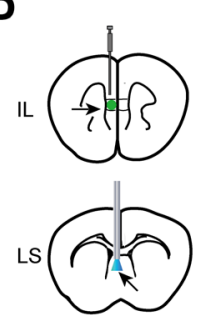

I

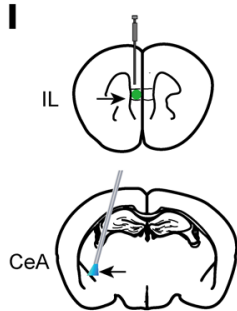

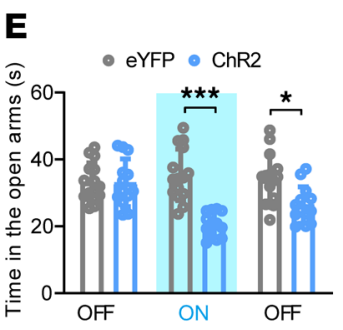

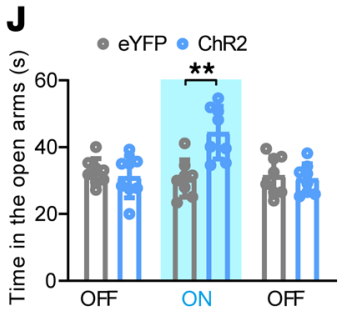

$\mathbf{F}$
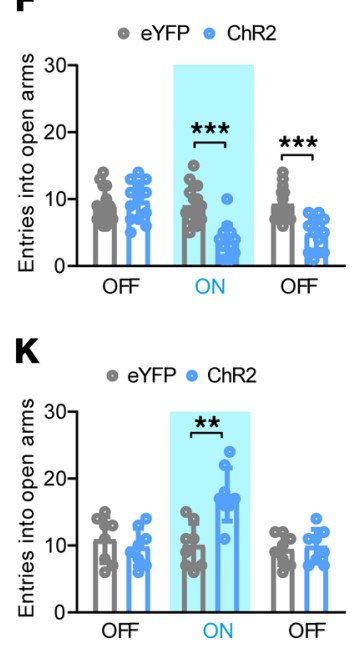

G

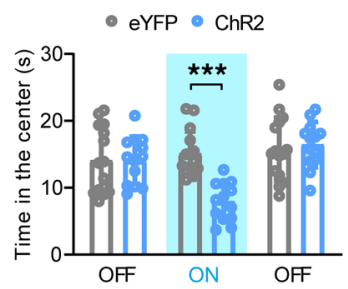

$\mathbf{L}$

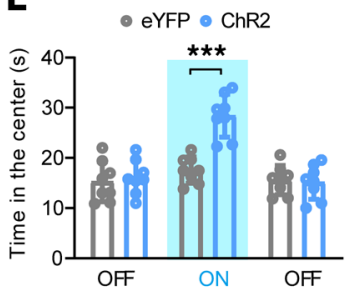

H

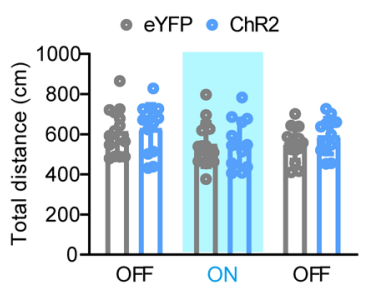

M



Figure 2. Distinct IL outputs modulate opposite anxiety-related behaviors. (A and B) Confocal images of coronal sections showing IL terminals in the LS (A), BLA, and CeA (B). Scale bars: $200 \mu \mathrm{m}$. (C) Experimental design to investigate the behavioral impact of optogenetic activation of IL-LS or IL-CeA projections. (D) eYFP and ChR2 mice expressing eYFP or ChR2-eYFP, respectively, in the IL with optic fibers above the LS. $n=15$ eYFP mic; $n=13$ ChR2 mice. $(\mathbf{E}-\mathbf{H})$ Blue light decreased the exploration of open arms $\left[\mathbf{E}, F_{\text {interaction }}(2,52)=8.447, P=0.0007\right]$, as well as the probability of open-arm entry $[\mathbf{F}$, $\left.F_{\text {interaction }}(2,52)=11.35, P=0.0002\right]$ and center exploration in the open field $\left[\mathbf{G}, F_{\text {interaction }}(2,52)=11.90, P<0.0001\right]$, without altering locomotion $\left[\mathbf{H}, F_{\text {int }}\right.$ $(2,52)=0.1149, P=0.8917]$. (I-M) Same as in $\mathbf{D}-\mathbf{H}$, but for IL-CeA projections. $n=8$ eYFP mice; $n=8$ ChR2 mice. Yellow light increased the exploration of open arms [J, $\left.F_{\text {interaction }}(2,28)=7.20, P=0.0030\right]$, resulted in a higher probability of open-arm entry $\left[K, F_{\text {interaction }}(2,28)=7.264, P=0.0029\right]$, increased center exploration in the open field $\left[\mathbf{L}, F_{\text {interaction }}(2,28)=12.18, P=0.0002\right]$, and did not alter locomotion $\left[M, F_{\text {interaction }}(2,28)=0.3525, P=0.7060\right]$. ${ }^{*} P<0.05, * * P<$ 0.01 , and ${ }^{* * *} P<0.001$, by 2 -way, repeated-measures ANOVA with Bonferroni's post hoc analysis $(\mathbf{E}-\mathbf{H}$ and $\mathbf{J}-\mathbf{M})$. Data are presented as the mean $\pm \mathrm{SEM}$. See Supplemental Table 1 for statistical details. 
$\mathbf{A}$

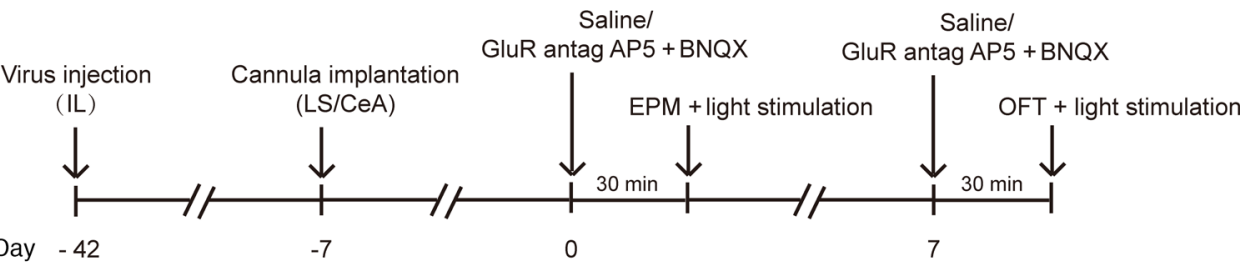

B

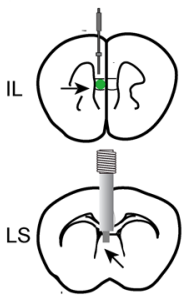

C Saline $\cdot A P 5+N B Q X$
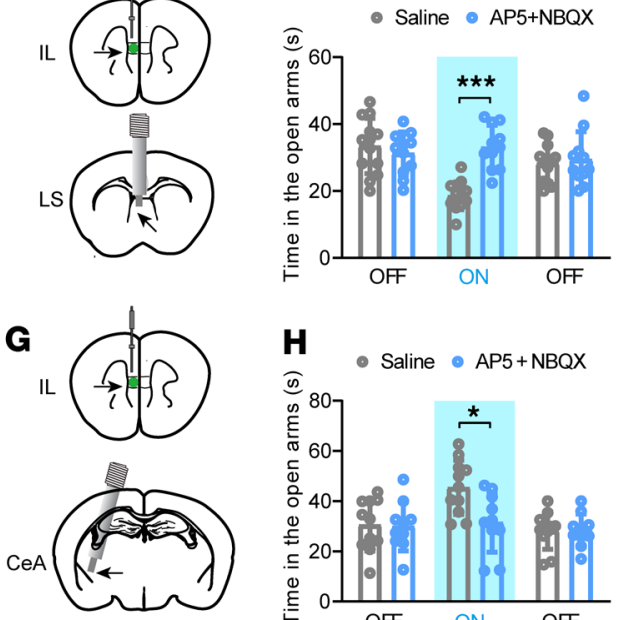

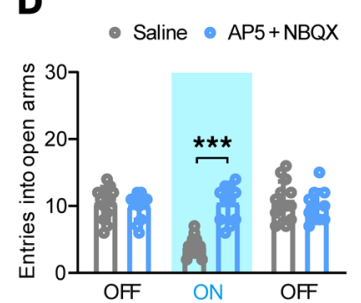

I

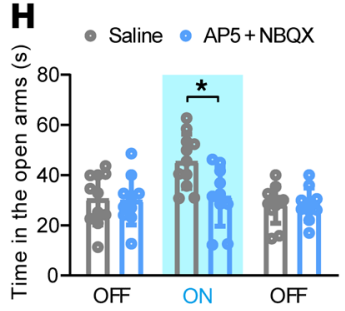

D

E
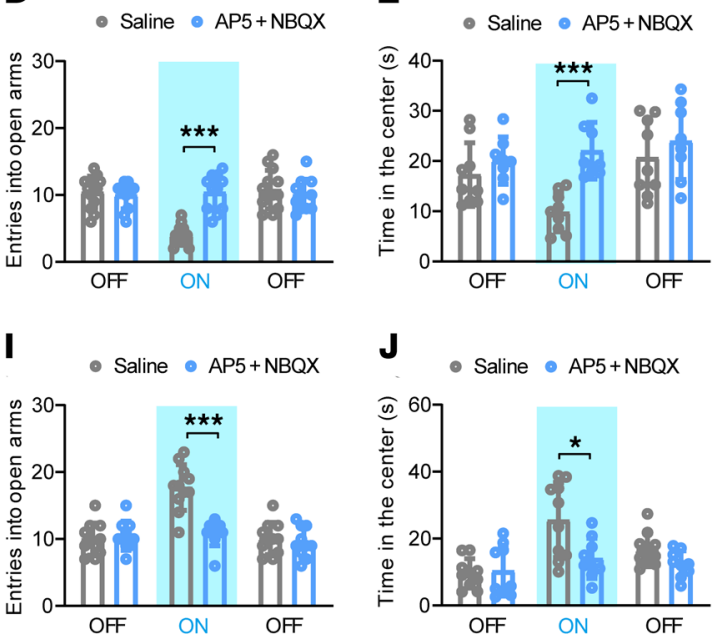

$\mathbf{F}$

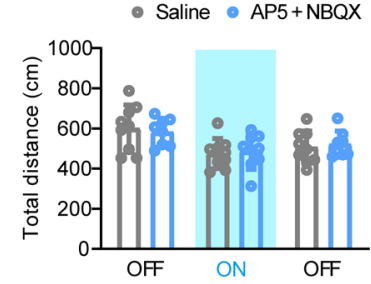

K

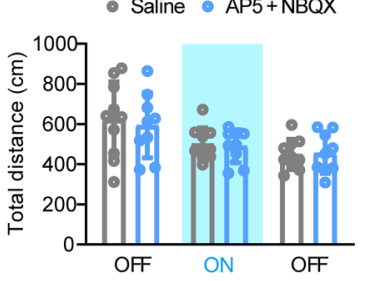

Figure 3. Glutamatergic IL inputs into the LS or CeA modulate anxiety-related behaviors. (A) Experimental paradigm. Experiments were performed 7 or 8 weeks after AAV2-CaMKIl $\alpha$-ChR2-eYFP injection. Thirty minutes before the behavioral assays and laser stimulation, glutamate receptor antagonists (GluR antag: AP5 + NBQX) or saline were unilaterally infused locally into the LS or CeA using the same guide cannula that delivered light via an optical fiber. (B) Mice expressing ChR2 in the IL were treated with saline or AP5 plus NBQX via cannulas above the LS. (C-F) Relative to saline injections, AP5 plus NBQX injections into the LS blocked the light-induced decreases in open-arm exploration on the EPM test $\left[\mathbf{C}, F_{\text {interaction }}(2,44)=12.57, P<0.0001 ; \mathbf{D}, F_{\text {interaction }}(2,44)\right.$ $=17.71, P=0.000]$ and center exploration in the open field $\left[E, F_{\text {interaction }}(2,30)=3.392, P=0.0470\right]$. Neither light stimulation nor injection of AP5 plus NBQX altered the total distance traveled $\left[\mathbf{F}, F_{\text {interaction }}(2,30)=0.2107, P=0.8112\right]$. (G-K) Same as in $\mathbf{B}-\mathbf{F}$, but for IL-CeA projections. After intra-CeA glutamate receptor blockade, photoactivation of ChR2-expressing IL terminals in the CeA failed to increase open-arm exploration $\left[\mathbf{H}, F_{\text {interaction }}(2,38)=3.100, P=\right.$ $\left.0.0566 ; \mathbf{I}, F_{\text {interaction }}(2,38)=13.84, P<0.0001\right]$, center exploration in the open field [J, $\left.F_{\text {interaction }}(2,34)=7.009, P=0.0028\right]$, or locomotion $\left[\mathbf{K}, F_{\text {interaction }}(2,34)=\right.$ 0.08985, $P=0.7863$. $n=11$ and $n=13$ mice for $\mathbf{C}$ and $\mathbf{D} ; n=9$ and $n=8$ mice for $\mathbf{E}$ and $\mathbf{F} ; n=11$ and $n=10$ mice for $\mathbf{H}$ and $\mathbf{I} ; n=10$ and $n=9$ mice for $\mathbf{J}$ and $\mathbf{K}$. ${ }^{*} P<0.05$ and ${ }^{* * *} P<0.001$., by 2 -way, repeated-measures ANOVA with Bonferroni's post hoc analysis $(\mathbf{C}-\mathbf{F}$ and $\mathbf{H}-\mathbf{K})$. Data are presented as the mean \pm SEM. See Supplemental Table 1 for statistical details.

2 distinct colors into the LS and CeA (Figure 4, O and P), we found that there were more LS- than CeA-projecting cells in the IL (Figure 4, Q and R), which was consistent with the retrobead and CAVCre labeling experiments (Figure 4, A-F). Furthermore, LS- and CeA-projecting cells shared little overlap (Figure 4, Q and R), indicating that LS- and CeA-projecting IL neurons are anatomically distinct subpopulations.

Inhibition of IL-LS and IL-CeA projections has opposite effects on anxiety-like behavior. To further confirm that IL-LS and IL-CeA projections bidirectionally modulated anxiety-related behaviors, we optogenetically inhibited these 2 circuits. We induced expression of eNpHR or eYFP alone in the IL and bilaterally implanted optical fibers above the LS or the CeA prior to testing in the anxiety assays (Figure 5A and Supplemental Figure 7). Relative to the eYFP controls, the animals in the eNpHR IL-LS group showed increased open-arm exploration (Figure 5B) and a higher probability of open-arm entry (Figure 5C) in the EPM test, as well as an increase in center exploration time (Figure 5D) in the OFT during the ON epoch, reflecting reductions in anxiety-related behaviors. In contrast, relative to the eYFP controls, the animals in the eNpHR IL-CeA group showed decreased open-arm exploration
(Figure 5B) and a lower probability of open-arm entry (Figure 5C) on the EPM test, as well as a decrease in center exploration time (Figure 5D) in the OFT during the $\mathrm{ON}$ epoch, reflecting anxiogenic effects. However, there were no changes in the distance traveled by mice in either group (Figure $5 \mathrm{E}$ ).

As previous reports have revealed some paradoxical effects of eNpHR when activated in presynaptic boutons (46), we next used inhibitory designer receptors exclusively activated by designer drugs (DREADD) (AAV-hM4DGi) to silence the IL-LS and IL-CeA pathways (47). Mice were transduced with hM4DGi in experimental animals and mCherry in controls in the IL and implanted with bilateral guide cannulas to either the LS or CeA (Supplemental Figure 8, A, C, and D). Clozapine- $\mathrm{N}$-oxide (CNO) was infused into the above regions 30 minutes prior to the behavioral tests, after which the mice were allowed 5 minutes of free exploration in the EPM or open-field chamber (Figure 5F). hM4DGi-mCherry was expressed strictly in the IL (Supplemental Figure 8A). Whole-cell recordings from brain slices revealed that hM4DGi-mCherry ${ }^{+}$ neurons were hyperpolarized by CNO application (Supplemental Figure 8B). Consistent with optogenetic inhibition, pharmacogenetic inhibition of the IL-LS circuit resulted in significantly 
A

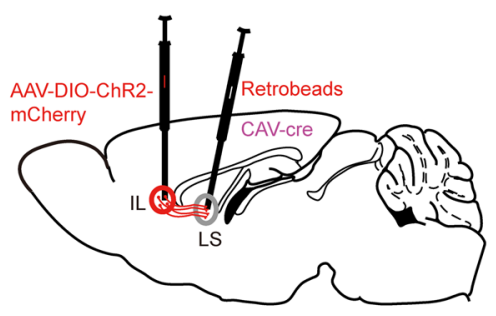

D

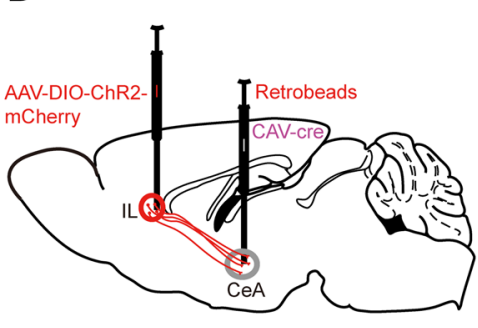

E
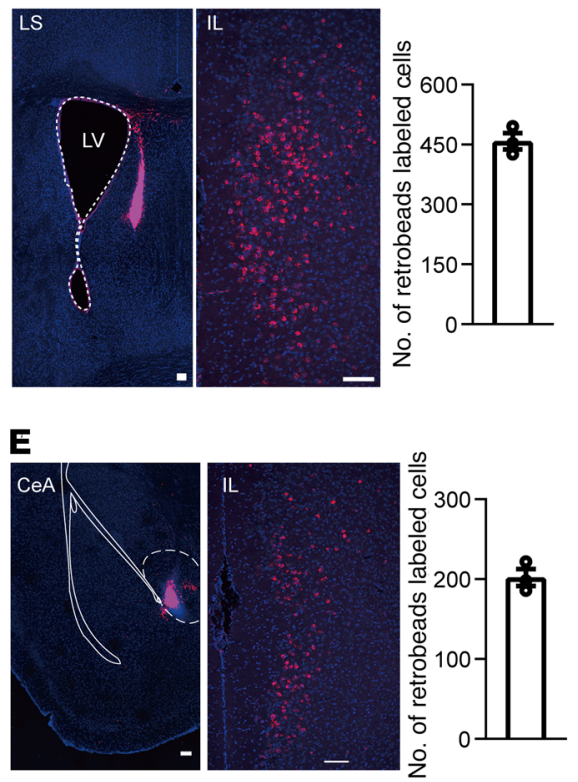

C
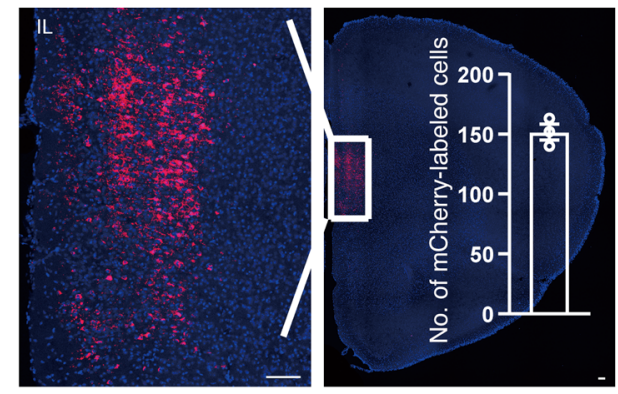

$\mathbf{F}$
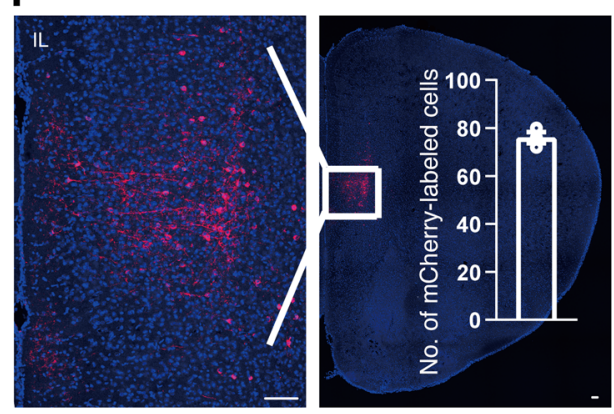

G

H
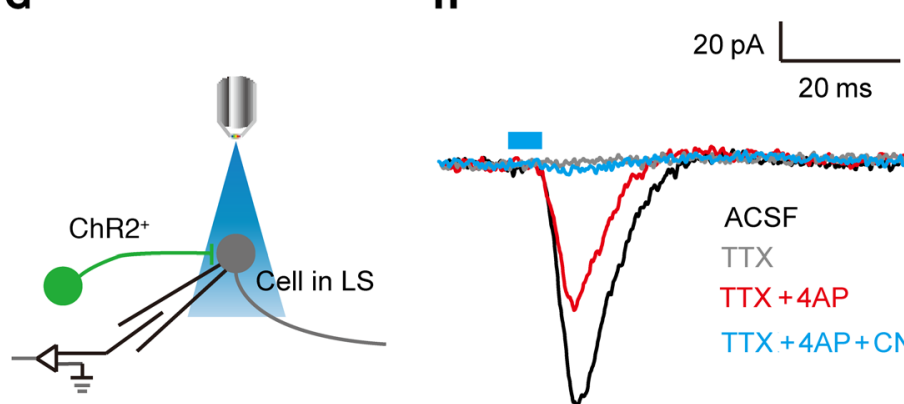

$\mathbf{K}$

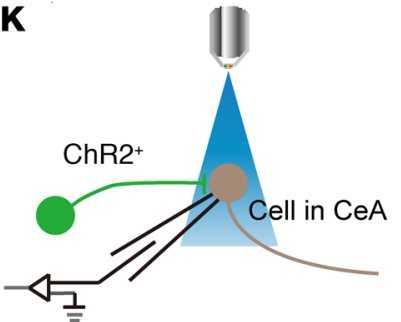

$\mathbf{L}$

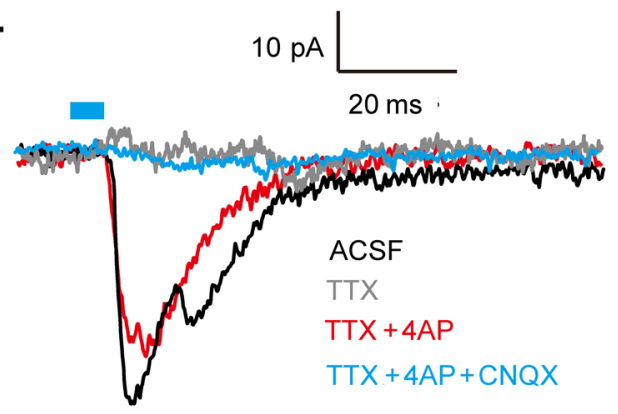

I

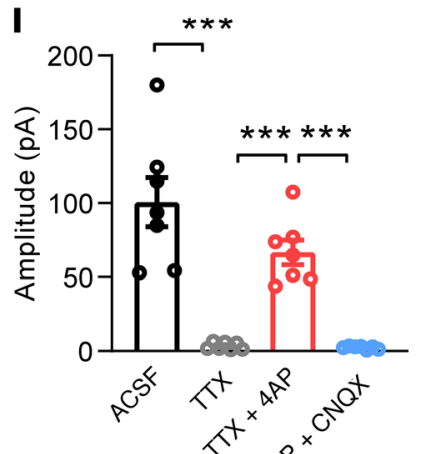

J

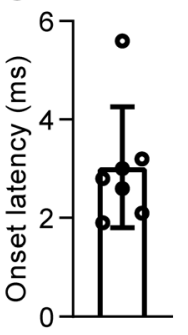

$T T X+4 A P+C N Q X$
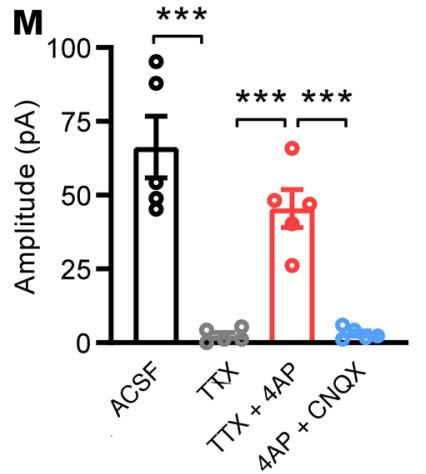

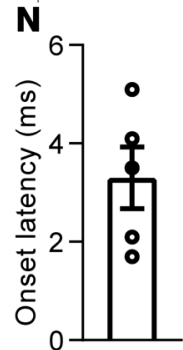

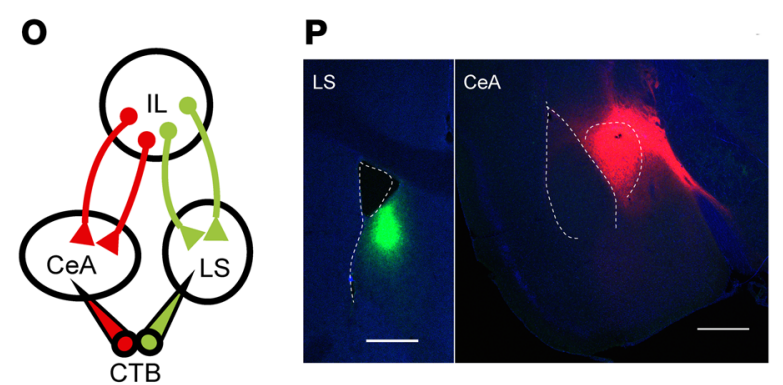

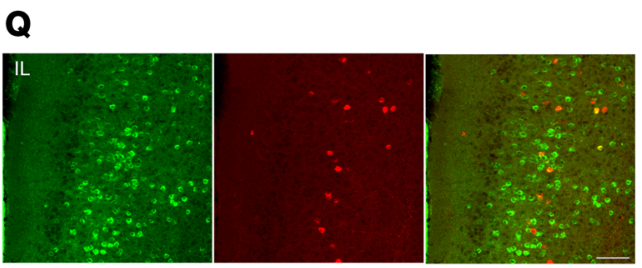

R

LS- vs CeA-projection

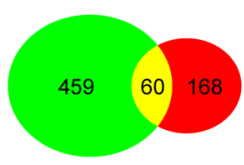


Figure 4. Monosynaptic and functional IL inputs into the LS or CeA. (A and D) Experimental scheme. Two methods were used: injection of retrobeads into the LS (A) or CeA (D) and injection of CAV-Cre virus into the LS (A) or CeA (D) and AAV-DIO-ChR2-Cherry virus into the IL (A and D). (B) Fluorescence images illustrating retrobead back-labeled LS-projecting IL neurons and graph indicating the total number of these neurons. $\mathrm{LV}$, lateral ventricle. (C) Same as in B, but for mCherry expression. (E) Same as in B, but for CeA-projecting IL neurons. (F) Same as in E, but for $\mathrm{mCherry} \mathrm{expression.} \mathrm{(G} \mathrm{and} \mathbf{K})$ Scheme for recording postsynaptic currents in the LS (G) or CeA (K) evoked by optogenetic activation of IL projections. ( $\mathbf{H}$ and $\mathbf{L}$ ) Overlay of light-triggered responses in the LS $(\mathbf{H})$ and CeA (L). (I and $\mathbf{M}$ ) Light-evoked postsynaptic currents were completely blocked by TTX and recovered by TTX plus 4AP, which were blocked by 6-cyano-7-nitroquinoxaline-2,3-dioneis (CNQX) [I, $n=7$ neurons from 2 mice, $F(3,24)=27.01, P<0.0001 ; \mathbf{M}, n=5$ neurons from 2 mice, $F(3,16)=$ 26.54, $P<0.0001$; 1-way ANOVA with Tukey's multiple-comparison test]. (J and $\mathbf{N}$ ) Onset latencies for the LS (J) and CeA (N). (0) Experimental scheme showing that CTB was injected into 2 targets of IL neurons, the LS and the CeA. (P) Fluorescence images illustrating CTB targeted to the LS (CTB488, green) and the CeA (CTB555, red). (Q) Coronal section of the IL labeled with CTB in green (LS) and red (CeA). (R) Number of IL neurons labeled with CTB. LS-projecting IL neurons, $n=519 \pm 13$ cells (average from 3 mice; 459 [green circle] + 60 [yellow overlap] = 519); CeA-projecting IL neurons, $n=228 \pm 11$ cells (average from 3 mice; 168 [red circle] + 60 [yellow overlap] = 228). Scale bars: $100 \mu \mathrm{m}(\mathbf{B}, \mathbf{C}, \mathbf{E}, \mathbf{F}$, and Q) and 500 $\mu \mathrm{m}(\mathbf{P}) .{ }^{* *} P<0.001$, by 1-way ANOVA with Tukey's multiple-comparison test (I and $\mathbf{M})$. Data are presented as the mean \pm SEM. See Supplemental Table 1 for statistical details.

increased open-arm exploration (Figure $5 G$ ) and a higher probability of open-arm entry (Figure 5H) in the EPM test and increased center exploration (Figure 5I) in the OFT. In contrast, pharmacogenetic inhibition of the IL-CeA circuit resulted in significantly reduced open-arm exploration (Figure $5 \mathrm{G}$ ) and a lower probability of open-arm entry (Figure $5 \mathrm{H}$ ) on the EPM test and decreased center exploration (Figure 5I) in the OFT with no effect on locomotion (Figure 5J). These results therefore support the hypothesis that IL-LS and IL-CeA projections bidirectionally modulate anxiety-related behaviors.

$I L-L S$ and IL-CeA projections regulate fear extinction in opposite directions. The IL has also been implicated in mediating fear conditioning based on physical, pharmacological, and genetic manipulations of this structure $(20,48-50)$. One study recently reported that real-time optogenetic manipulation of the IL modulates fear extinction in rats (31); thus, we revisited these experiments in mice and subsequently investigated downstream targets of the IL in the regulation of fear extinction.

We used 2 strategies to alter IL activity: silencing with eNpHR and activation with ChR2 (Supplemental Figure 9, A and C). We conducted consecutive tests of cued fear conditioning with the eNpHR mice, the ChR2 mice, and the eYFP control mice by presenting a 30-second auditory tone (conditioned stimulus [CS]) that coterminated with a 1-second footshock for 4 trials. As training progressed, each group of mice became increasingly immobilized (freezing) during the tone and exhibited similar freezing levels during the conditioning phase (Supplemental Figure 9B). We then tested whether manipulation of IL neuronal activity affected fear memory retrieval on day 2 . We placed the mice in a test chamber that was distinct from the training chamber and delivered a continuous auditory tone in the absence of a footshock. Laser illumination was initiated 1 second (for ChR2 mice) or $10 \mathrm{sec}-$ onds (for eNpHR mice) before tone onset and sustained during the tone, given the long response latencies observed in some IL neurons following laser illumination (31). We found that ChR2 activation of IL neurons significantly reduced freezing, whereas eNpHR inhibition had no effect on freezing (Supplemental Figure 9B). Following behavioral protocols for testing fear extinction (51, 52) on day 3 , the mice were placed in a test chamber and received 10 trials of the CS at random intervals without a footshock. The mice in the stimulation groups received the same illumination paradigm as on day 2. The eYFP control mice showed a normal response pattern: freezing responses that decreased gradually within a session and became fully extinguished after 10 CS stimulations. Replicating our finding above, optogenetic activation of IL neurons reduced conditioned freezing in all trials (Supplemental Figure 9B). Notably, optogenetic inhibition of IL neurons had no effect on the levels of freezing compared with the levels of freezing in the eYFP control mice throughout the test (Supplemental Figure 9B). On day 4, we tested extinction retrieval with a 180 -second continuous auditory tone without any optical stimulation. We found that ChR2-activated mice continued to show reduced freezing, while mice that received IL silencing the previous day showed impaired extinction retrieval (Supplemental Figure 9B). These data suggested that IL activity during extinction tones was necessary for the formation of extinction memory, in accordance with previous reports (31).

To explore whether distinct projections of the IL mediate top-down control of fear conditioning, we first activated IL axons in the LS and CeA. We induced expression of ChR2 in the experimental animals and expression of eYFP alone in the control animals in the IL and unilaterally implanted an optical fiber over either the CeA or the LS in the ipsilateral hemisphere prior to testing in the fear assays (Figure 6A and Supplemental Figure 10A). Each group of animals showed a similar increase in freezing during the conditioning phase and similar levels of fear retrieval on the following day under blue light stimulation (Figure 6B). In the extinction sessions, IL-LS activation attenuated fear extinction, whereas IL-CeA activation enhanced fear extinction (Figure 6B). When tested with a continuous 180 -second tone on day 4, the ChR2-LS mice showed higher freezing levels, whereas the ChR2-CeA mice showed lower freezing levels (Figure 6B) without light stimulation. These data suggested the involvement of the IL-CeA and IL-LS pathways in fear extinction.

We next tested the effects of pharmacogenetic inhibition of IL axons in the LS or CeA on fear retrieval and extinction. hM4DGi or mCherry alone was delivered into the IL along with placement of a guide cannula above either the LS or CeA prior to testing in the fear-conditioning assays (Figure 6C and Supplemental Figure 10B). We induced fear responses by conditioning mice with 4 tone-footshock pairs. Local infusion of CNO had no effect on fear retrieval on day 2 (Figure 6D). During the extinction phase the following day, the freezing levels in the hM4DGi IL-CeA group receiving CNO were initially similar to those in the control mice but decayed more slowly, resulting in significantly higher freezing ratios (Figure 6D). In contrast, the hM4DGi-IL-LS group receiving CNO showed similar levels of freezing at the beginning but enhanced fear extinction afterwards (Figure 6D). In the extinction 
A

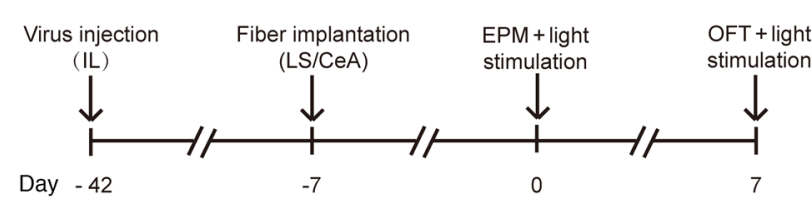

C
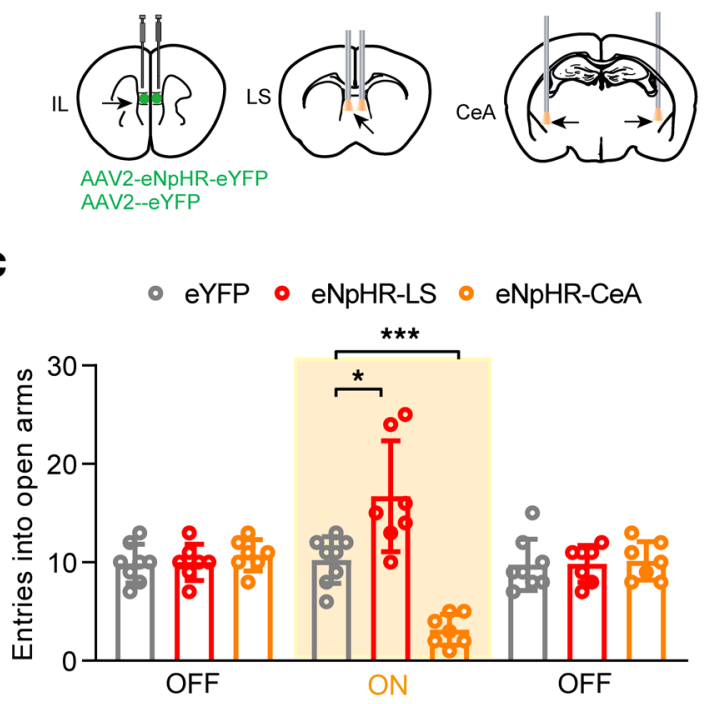

$\mathbf{E}$

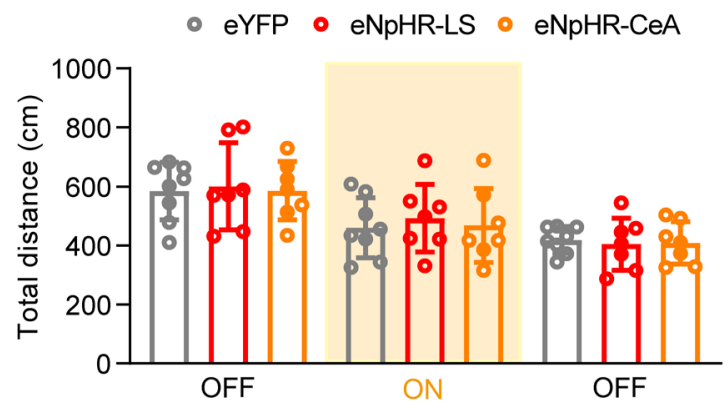

G

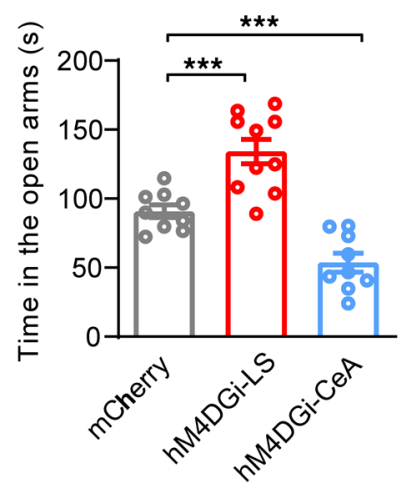

B

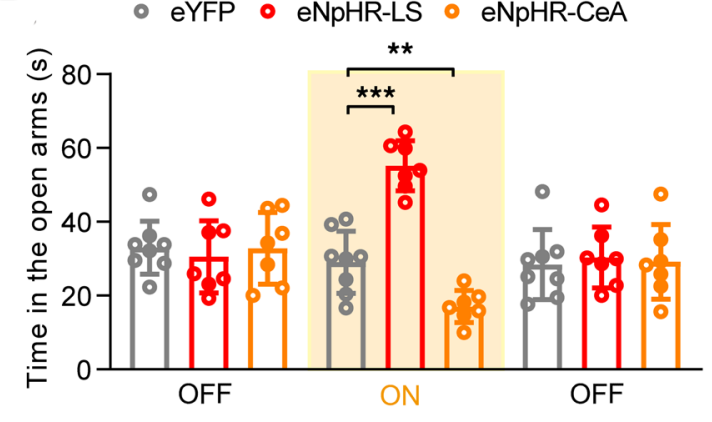

D
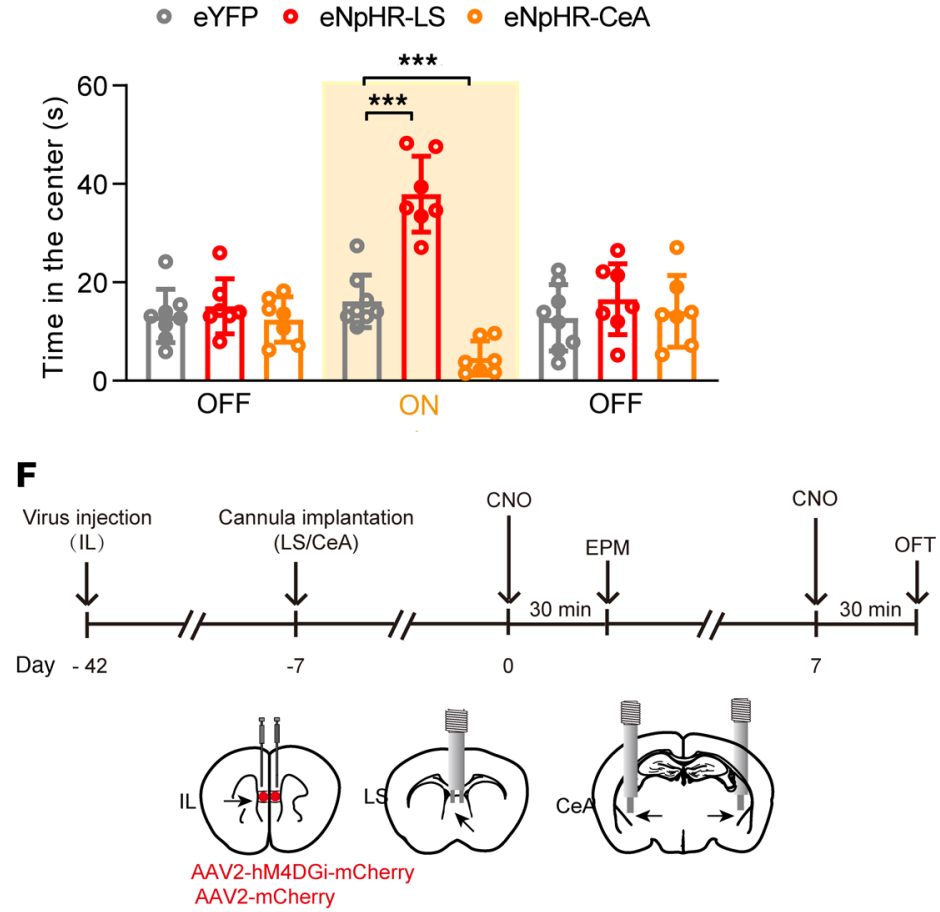

J

Figure 5. IL-LS and IL-CeA inhibition shows opposite effects on anxietyrelated behaviors. (A) Experimental paradigm for optogenetic inhibition. Neurons in the IL were transduced with either NpHR-eYFP or eYFP. Yellow light was delivered via bilateral optical fibers implanted into the LS or CeA after 6-7 weeks of viral incubation. (B-D) eNpHR IL-LS mice, compared with eYFP mice, showed increased open-arm exploration [B, $F$ $(4,38)=15.69, P<0.0001]$ and a higher probability of open-arm entry [C, $\left.F_{\text {interaction }}(4,38)=17.89, P<0.0001\right]$ in the EPM test and increased center exploration time $\left[\mathbf{D}, F_{\text {interaction }}(4,38)=18.38, P<0.0001\right]$ in the OFT during the illumination epoch, whereas yellow light induced opposite effects on the NpHR-IL-CeA mice. $n=8$ eYFP mice; $n=7$ eNpHR-LS mice; $n=7$ eNpHR-CeA mice. (E) total distance traveled. (F) Experimental paradigm for pharmacogenetic inhibition. Neurons in the IL were transduced with either hM4DGi-mCherry or mCherry. CNO was delivered via bilateral guide cannula implanted into the LS or CeA 30 minutes before the behavioral assays, after 6 to 7 weeks of viral incubation. (G-I) hM4DGi IL-LS mice, compared with mCherry mice, showed an increase in both open-arm exploration in the EPM test $[\mathbf{G}, F(2,25)=36.92, P<0.0001 ; \mathbf{H}, F(2,25)=$ $32.10, P<0.0001]$ and center exploration in the OFT $[I, F(2,25)=80.83$, $P<0.0001]$ after CNO infusion, whereas CNO induced opposite effects on the hM4DGi IL-CeA mice. $n=9$ eYFP mice; $n=10$ eNpHR-LS mice; $n$ $=9$ eNpHR-CeA mice. (J) Total distance traveled. ${ }^{*} P<0.05,{ }^{*} P<0.01$, and ${ }^{* *} P<0.001$, by 2-way, repeated-measures ANOVA with Dunnett's multiple-comparison test (B-E) and 1-way ANOVA with Dunnett's multiple-comparison test (G-J). Data are presented as the mean $\pm \mathrm{SEM}$. See Supplemental Table 1 for statistical details.

retrieval session on day 4, a continuous 180-second tone elicited significantly higher freezing levels in the hM4DGiIL-CeA mice and lower levels in the hM4DGiIL-LS mice (Figure 6D) in the absence of CNO.

Taken together, these results provide evidence that the IL-CeA and IL-LS circuits are critical for controlling fear extinction but with opposite actions.

Activation of CeL- and CeM-projecting IL neurons differentially regulates fear-related behaviors. The CeA contains the lateral subdivision of the central amygdala $(\mathrm{CeL})$, the medial central amygdala (CeM), and the capsular central amygdala (53). Neuronal activity in the CeL is required for fear acquisition, whereas conditioned fear responses are driven by output neurons in the CeM (36). Functional circuit analysis revealed that CeM output neurons are under inhibitory control from the CeL $(36,37)$. We next explored whether specific activation of IL neurons projecting to distinct subdivisions of the CeA differentially modulate anxiety and fear. We targeted CeL- and CeM-projecting IL neurons by injecting CAV-Cre into either the $\mathrm{CeL}$ or the CeM and directing AAV-DIO-ChR2-mCherry as well as optical fibers into the IL (Figure 7, A-D, and Supplemental Figure 11, A-D). Putative starter cells in the IL were labeled, as defined by neurons that expressed mCherry (Figure 7, C and E). Activation of CeL-projecting cells increased openarm exploration (Figure 7F) and a higher probability of openarm entry in the EPM test (Figure $7 \mathrm{G}$ ), and increased center exploration in the OFT (Figure 7H) during the ON epoch without affecting locomotion (Figure 7I), reflecting reductions in anxiety-related behaviors. In addition, activation of CeM-projecting cells increased center exploration in the OFT and had no effects on behavior in the EPM test (Figure 7, F-I). Notably, activation of CeM-projecting cells decreased freezing during extinction and extinction retrieval, whereas activation of CeL-projecting cells had no effect on behavior (Figure 7J). These results suggested that stimulation of the IL-CeM mainly accounted for the behavioral effects seen following IL-CeA activation. CeL contains a GABAergic subpopulation marked by protein kinase $\mathrm{C}-\delta(\mathrm{PKC}-\delta)$. These neurons inhibit output neurons in the CeM and make reciprocal inhibitory connections with $\mathrm{PKC}-\delta^{-}$neurons within the $\operatorname{CeL}(37,38)$. We further explored which kinds of neurons are recruited in IL-CeL activation. ChR2-IL-CeL mice received blue light in the IL (Supplemental Figure 11E). Accordingly, CeL-projecting IL activation induced c-Fos expression in both $\mathrm{PKC}-\delta^{+}$and $\mathrm{PKC}-\delta^{-}$neurons at a similar ratio (Supplemental Figure 11, F and G), implying that the absence of effects on fear extinction following stimulation of CeL-projecting cells may have been due to the simultaneous activation of these 2 types of neurons.

Activation of the IL-CeA circuit alleviates stress-induced anxiety. Anxiolysis produced by IL-CeA circuit activation raised the possibility of top-down modulation of behavior elicited by pathological conditions. To verify this hypothesis, we tested whether activation of the IL-CeA pathway could reverse stress-induced anxiety-like behaviors. Virus was injected into the IL cortex along with implantation of an optical fiber over the CeA (Supplemental Figure 12). Mice were restrained for 1 hour each day on 3 consecutive days and then underwent behavioral tests (Figure 8A). Restraint stress increased the avoidance of open arms (Figure 8, B and C) and the center zone (Figure 8D) for the stressed mice compared with the naive control animals without affecting locomotor activity (Figure $8 \mathrm{E}$ ). These data showed that exposure to restraint stress enhanced anxiety-like behaviors in mice. IL-CeA activation in the stress-ChR2 group compared with the stress-eYFP group reversed the high anxiety-like behaviors revealed by the EPM test (Figure 8, B and C), and a similar phenomenon was observed in the OFT (Figure 8D) without affecting locomotor activity (Figure 8E). Together, these results suggested that activation of the IL-CeA pathway was sufficient to reverse stress-induced anxiety.

\section{Discussion}

The role of the IL and its innervated subcortical regions in regulating anxiety and fear has been studied separately, but precise direct targets for IL projections in the top-down control of these moods have not been identified. Here, we define 2 top-down behavioral regulation pathways. We identified LS as the direct target of the IL that enhances fear-related freezing and anxiety states, whereas the IL-CeA pathway was sufficient and necessary for anxiolysis.

Under physiological conditions, the mPFC exerted inhibitory top-down control over amygdala activity, limiting its output and thus preventing inappropriate emotional expression (54-57). We consistently uncovered such an inhibitory circuit - the IL-CeA projection - as inhibition of this circuit was anxiogenic, indicating that it is required for restraining anxiety in physiological situations. On the other hand, when animals are exposed to anxietyprovoking environments such as the EPM and OFT, mPFC neurons represent anxiety-related features, and this representation is used by the animal to guide anxiety-related behavior $(58,59)$. In line with this, we found an anxiogenic circuit - the IL-LS pro- 
A

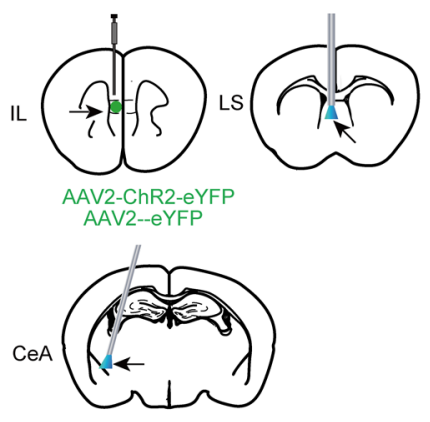

B

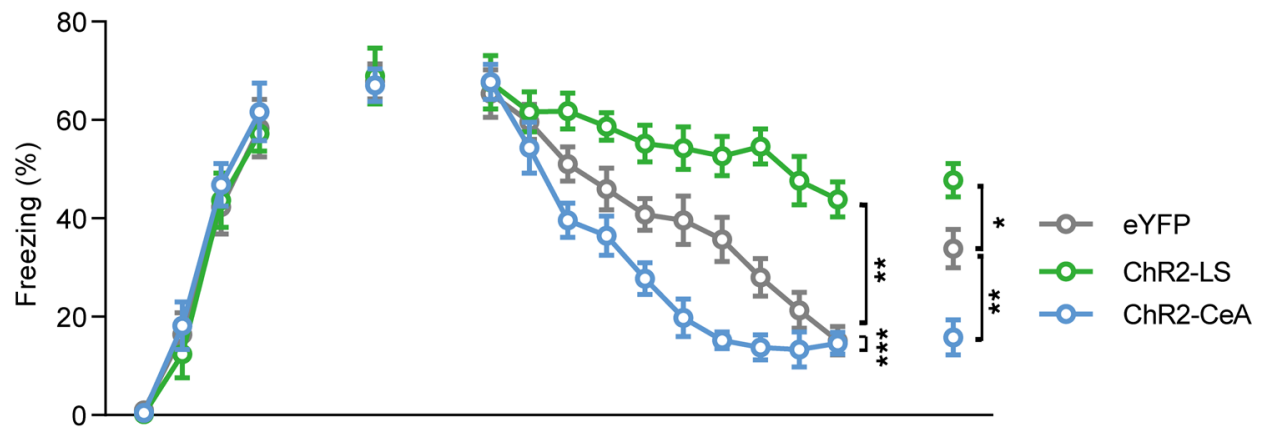

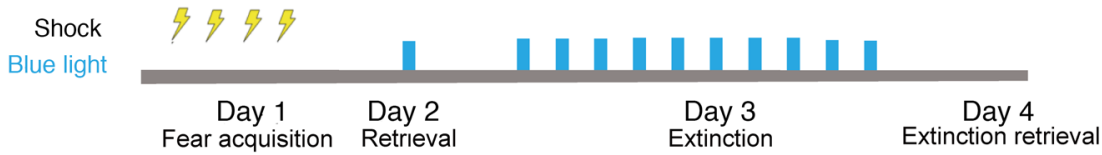

C

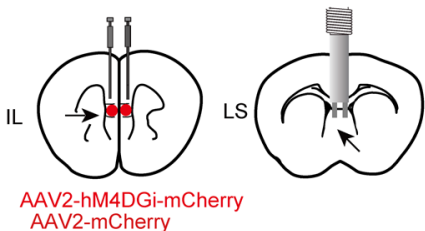

D

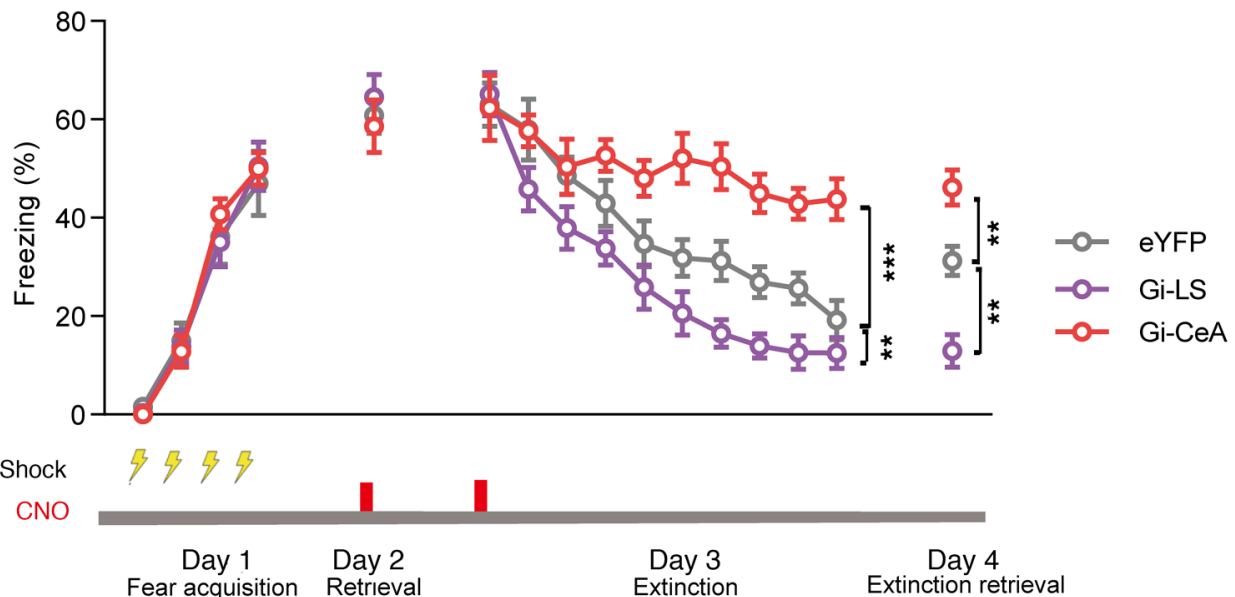

Figure 6. IL-LS and IL-CeA circuits modulate fear extinction in the opposite direction. (A) Scheme of mice expressing ChR2 or eYFP in the IL with optic fibers above the LS or CeA. (B) Effects of optogenetic activation of IL-LS (ChR2-LS) and IL-CeA (ChR2-CeA) projections on fear retrieval, extinction, and extinction retrieval. Blue light was delivered on day 2 and day 3. Mice showed similar fear retrieval in each group $[F(2,23)=0.04646, P=0.9547]$. ChR2-LS mice froze more than did eYFP control mice, while ChR2-CeA mice froze less during fear extinction $\left[F_{\text {interaction }}(18,207)=3.54, P<0.0001\right]$ and extinction retrieval $[F(2,23)=17.23, P<0.0001]$. $n=10$ eYFP mice; $n=8$ ChrR2-LS mice; $n=8$ ChR2-CeA mice. (C) Scheme of mice expressing hM4DGi or mCherry in the IL with a cannula above the LS or CeA. (D) Effects of pharmacogenetic inhibition of IL-LS (hM4DGi-LS [Gi-LS]) and IL-CeA (hM4DGi-CeA [Gi-CeA]) projections on fear retrieval, extinction, and extinction retrieval. CNO was delivered 30 minutes before fear retrieval and extinction. Mice showed similar degrees of fear retrieval in each group $[F(2,25)=0.4312, P=0.6545]$. hM4DGi-LS mice froze less than did mCherry control mice, whereas hM4DGi-CeA mice froze more during fear extinction $\left[F_{\text {interaction }}(18,230)=3.336, P<0.0001\right]$ and extinction retrieval $[F(2,25)=25.88, P<0.0001] . n=9$ mCherry mice; $n=10$ hM4DCi-LS mice; $n=9$ mice hM4DCi-CeA mice. ${ }^{*} P<0.05$, ${ }^{* *} P<0.01$, and ${ }^{* *} P<0.001$, by 1 -way ANOVA with Dunnett's multiple-comparison test for fear retrieval and extinction retrieval and 2-way, repeated-measures ANOVA with Dunnett's multiple-comparison test for fear extinction. Data are presented as the mean \pm SEM. See Supplemental Table 1 for statistical details.

jection - as inhibition of this circuit was anxiolytic. Thus, our findings reveal that there are 2 antagonized top-down control circuits in the MPFC for the modulation of anxiety states with high efficiency and precision.

Particular intrinsic, homeostatic, or counterbalancing elements orchestrate behavioral states in animals with highly diverse repertoires of internal states and adaptations to the environment $(43,60,61)$. Our findings suggest that IL control of anxiety is mediated by cooperation between LS- and CeAprojecting cell activities. The fact that LS- and CeA-projecting IL neurons are intermingled in a salt-and-pepper-like manner, rather than being anatomically segregated, may help to identify local interactions important for rapid behavioral switching. It remains to be determined how defined populations of IL projection neurons are integrated into local circuitry and which cell types they contact in their long-range target structures. Notably, the $\mathrm{mPFC}$ acts as a site of convergence, integrating inputs from brain structures, including amygdala/hippocampal inputs that regulate fear and anxiety (62-64). Eventually, an integrated understanding of functional circuit organization on both local and global scales will be required to determine how complex behaviors emerge and how they are modified by experience, which would be an interesting topic for future studies.

In addition, our data showed that both IL and IL-LS activation produced anxiogenic effects that outlasted laser stimulation. The mechanism underlying this sustained effect on anxiety is cur- 
A

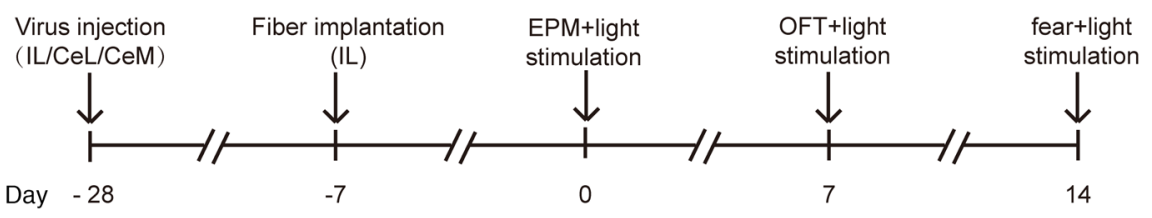

B

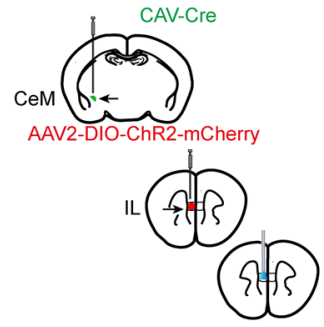

C

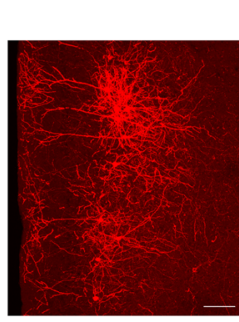

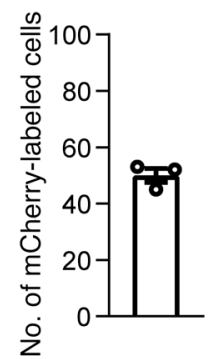

D

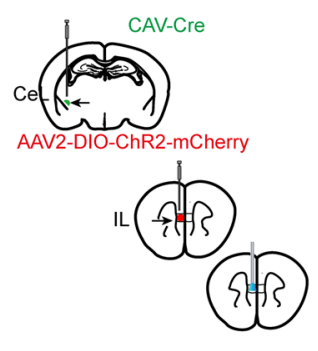

E

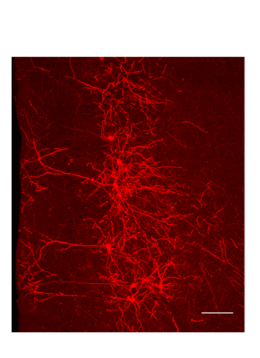

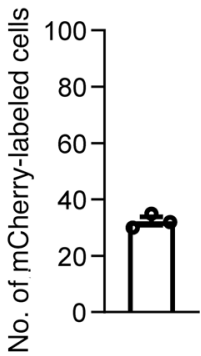

$\mathbf{F}$

- mChenry $\circ$ CeL-ChR2 $\odot$ CeM-ChR2

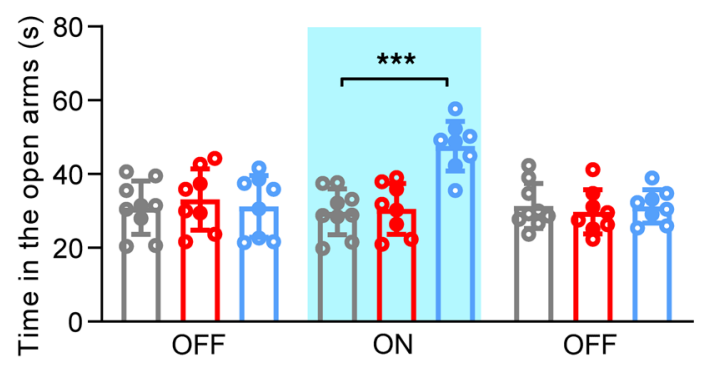

H

- mChenry • CeL-ChR2 • CeM-ChR2

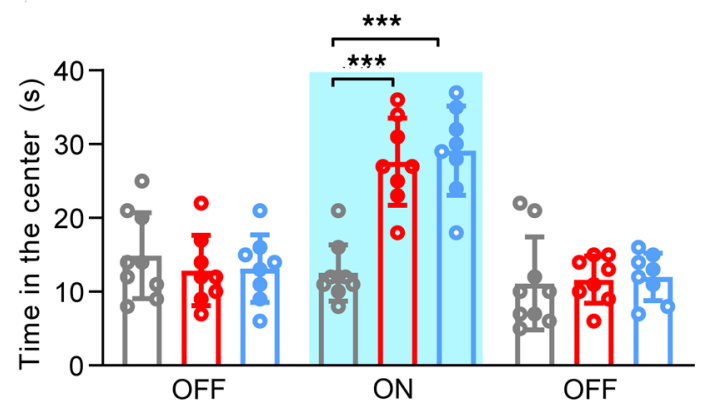

G

- mChenry ○ CeL-ChR2 • CeM-ChR2

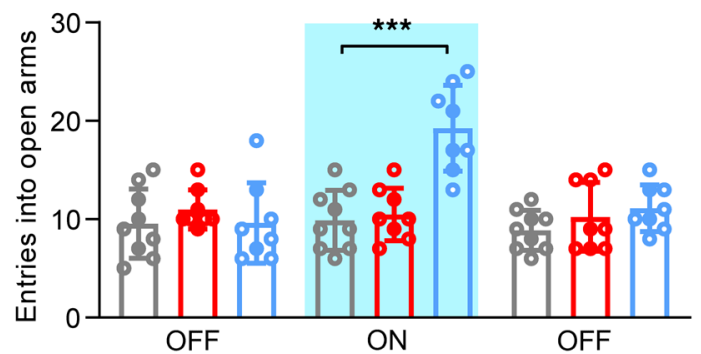

- mChenry • CeL-ChR2 • CeM-ChR2

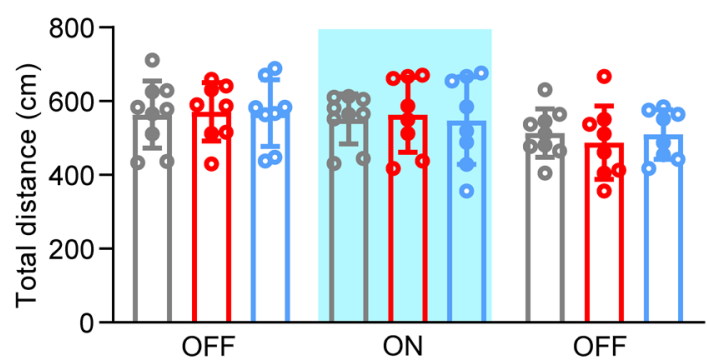

\section{J}

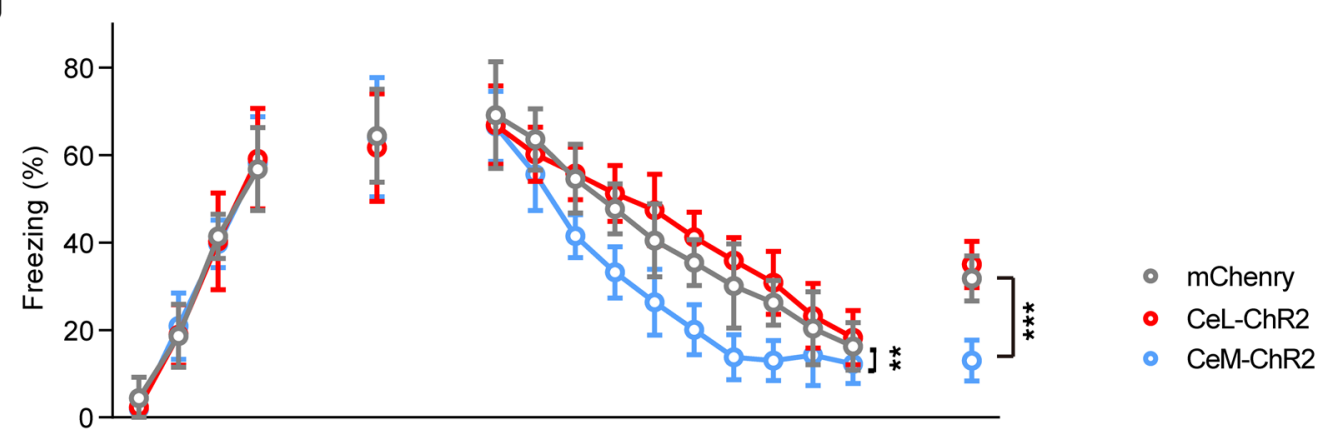

Shock

Blue light

3553

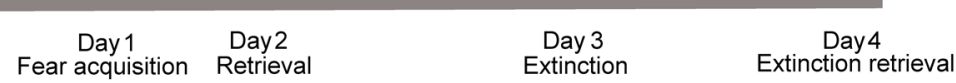


Figure 7. Activation of CeL- and CeM-projecting IL neurons differentially regulates fear-related behaviors. (A, B, and D) Experimental paradigm. Injection of CAV-Cre virus into the CeM (B) or CeL (D) and injection of AAV-DIO-ChR2-mCherry virus into the IL. Blue light was delivered via optical fibers implanted into the IL during the behavioral tests. (C) Image of mCherry expression in CeM-projecting IL neurons and graph showing the total number of CeM-projecting IL neurons. Scale bar: $200 \mu \mathrm{m}$. (E) Same as in D, but for CeL-projecting IL neurons. Scale bar: $200 \mu \mathrm{m}$. (F-H) CeM-ChR2-projecting mice showed increased open-arm exploration [F, $\left.F_{\text {interaction }}(4,38)=15.69, P<0.0001\right]$ and a higher probability of open-arm entry $\left[\mathbf{G}, F_{\text {interaction }}(4,38)=17.89, P<0.0001\right]$ in the EPM test, as well as an increase in center exploration time $\left[\mathbf{H}, F_{\text {interaction }}(4,38)=18.38, P<\right.$ 0.0001 ] in the OFT during the illumination epoch, whereas blue light only induced an increase in center exploration time $\left[\mathbf{H}, F_{\text {interaction }}(4,38)=18.38\right.$, $P<0.0001]$ in the OFT for CeL-ChR2-projecting mice. (I) Total distance traveled. (J) Effects of optogenetic activation of CeM-ChR2 and CeL-ChR2 mice on fear retrieval, extinction, and extinction retrieval. CeM-ChR2 mice froze less than did mCherry control mice, while CeL-ChR2 mice showed levels of freezing similar to those of mCherry control mice during fear extinction $\left[F_{\text {interaction }}(2,20)=13.51, P=0.0002\right]$ and extinction retrieval $[F(2,22)=45.12, P<0.0001] . n=9$ mCherry mice; $n=8$ CeM-ChR2 mice; $n$ $=8$ CeL-ChR2 mice. ${ }^{* *} P<0.001$, by 2 -way, repeated-measures ANOVA with Dunnett's multiple-comparison test $(\mathbf{F}-\mathbf{I})$ and 1 -way ANOVA with Dunnett's multiple-comparison test for fear retrieval and extinction retrieval and 2-way, repeated-measures ANOVA with Dunnett's multiple-comparison test for fear extinction (J). Data are presented as the mean \pm SEM. See Supplemental Table 1 for statistical details.

rently unknown but would also be an interesting topic for future studies. One possible mechanism for this phenomenon is synaptic plasticity, as the $20 \mathrm{~Hz}$ stimulation used in the present study has been reported to induce post-tetanic potentiation, which lasts for several minutes $(65,66)$. The LS has long been implicated in the control of stress responses and anxiety (67), and activation of LS Crfr2 ${ }^{+}$neurons promotes anxiety in a persistent manner (34). These results suggest that the IL-LS axis may be responsible for controlling persistent anxiety. It is interesting to note that 3 pathways have been found thus far to display persistent anxiogenesis (vBNST-VTA, LS-AHA, and IL-LS; refs. 34, 60). It remains to be seen whether persistent anxiolytic pathways exist or if persistence is a unique property of circuits that elevate anxiety.

The role of the septum in anxiety is complex. Inactivation studies indicate that different subdivisions of the septum (lateral, medial, or anterior) have opposite effects on anxiety-related behavior (68-71). The LS is also cellularly heterogeneous (72). Optogenetic activation of $\mathrm{Crfr}^{+}{ }^{+}$neurons in the LS exerts an anxiogenic effect (34), whereas electric stimulation of the LS and activation of LS cells receiving projections from the ventral hippocampus are anxiolytic $(73,74)$, and their identification probably depends on the differential genetic identification of cell populations. Our data show that IL fibers have strong inputs into the intermediate part of the LS (LSI) and moderate inputs into the dorsal part (LSD), while sparing the ventral part of the LS (LSV) across the anterior-posterior axis. Future studies should reveal the role of LS subdivisions and subpopulations in mediating top-down control of anxiety and fear.

Importantly, we note that direct DP activation produced anxiolytic effects, which may have counteracted the anxiogenic effects of IL activation, thereby leading to the lack of effects following whole vmPFC activation (13). This phenomenon suggests that attention should be given to specific local brain subregions with regard to treatment, such as deep brain stimulation.

Additionally, we show that activation of IL inputs into the CeA was sufficient to attenuate anxiety-like behaviors in both physiological conditions and stress-induced anxiety states. These data are consistent with reports implicating CeA involvement in anxiety $(35,75,76)$. The observation that selective illumination of specific IL-CeA terminals produced behavioral responses opposite of those seen with nonspecific illumination of all glutamatergic IL somata points to diversity in the IL population, in which more cells that project to the LS surpass the anxiolytic effects of IL-CeA projections. Alternatively, our observation suggests that LS-projecting cells are probably more active than CeAprojecting cells. In addition to providing insight into native anxiogenic and anxiolytic processes, these findings demonstrated that anxiety is continuously regulated by balanced antagonistic pathways between the IL-LS and IL-CeA pathways and that modulating the excitability of the IL-CeA projection may be beneficial for the treatment of anxiety disorders.

Optical activation of the IL neurons facilitated fear extinction and strengthened extinction retrieval the following day, consistent with previous electrical stimulation $(21,26)$ and optical and pharmacological manipulation $(27,31)$ studies. In addition, we found that IL-CeA and IL-LS circuits were critical for controlling fear extinction but with opposite actions. Importantly, the function of the IL-LS and IL-CeA pathways in regulating the extinction of fear memory was consistent with those regulating anxiety-like behaviors, as mentioned above.

Our data showed that neither optogenetic activation nor inhibition of IL-CeA terminals had any effect on fear retrieval, which is inconsistent with a previous report showing that inactivation of the CeA with $\mathrm{GABA}_{\mathrm{A}}$ agonists results in retrieval deficits (36). One explanation for this discrepancy may be that the CeA receives massive inputs to regulate fear retrieval other than those from the IL (77). The CeA contains at least 3 subnuclei (CeM, $\mathrm{CeL}$, and the capsular central amygdala; ref. 78) and multiple neuronal subtypes $(53,79)$. Depending on the activation of the CeA subdivision ( $\mathrm{CeL}$ or $\mathrm{CeM}$ ), as well as the specific cell population within the $\mathrm{CeL}\left(\mathrm{PKC}-\delta^{+}, \mathrm{SOM}^{+}\right)$, different fear-related responses could be induced (36-38). Optogenetic inhibition of $\mathrm{SOM}^{+} \mathrm{CeL}$ neurons, the majority of which are $\mathrm{PKC}-\delta^{-}$neurons (the $\mathrm{SOM}^{-}$ neurons are $\mathrm{PKC}-\delta^{+}$), suppresses fear retrieval (38). However, we found that direct stimulation of CeL-projecting IL neurons had no effect on fear retrieval. PKC- $\delta^{+}$and $\mathrm{PKC}-\delta^{-}$neurons in the $\mathrm{CeL}$ mutually inhibit each other $(37,38)$; therefore, simultaneous activation of both kinds of neurons may counteract their effects on fear retrieval. In line with this, we found that optogenetic activation of CeL-projecting IL neurons induced c-Fos expression in both $\mathrm{PKC}-\delta^{+}$and $\mathrm{PKC}-\delta^{-}$neurons at a similar ratio in the $\mathrm{CeL}$, with no alterations in fear-related behaviors, suggesting that IL-CeL PKC- $\delta^{+}$and IL-CeL PKC- $\delta^{-}$may play opposite roles. Future studies should investigate the IL inputs into CeL PKC- $\delta^{+}$and $\mathrm{PKC}-\delta^{-}$ neurons and the interaction of these neurons in the control of fear retrieval. Notably, activation of CeM- but not CeL-projecting IL neurons facilitated fear extinction, mimicking the effects of IL-CeA terminal stimulation. Since the CeM is under tonic inhibitory control from the $\mathrm{CeL}(36,37,80)$, the functional relationship 
A

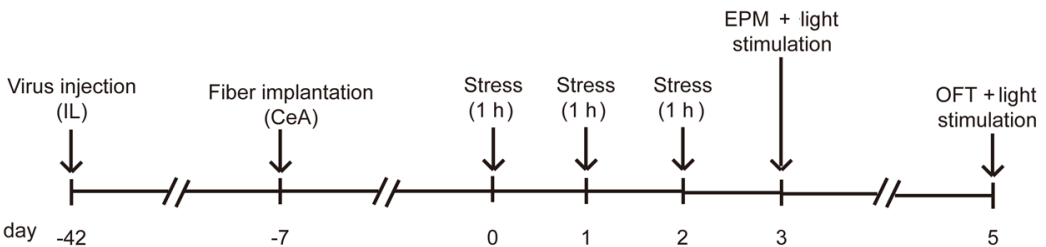

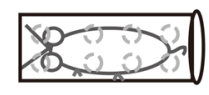

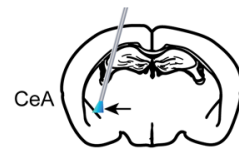

B

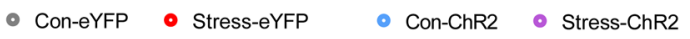

\section{(}
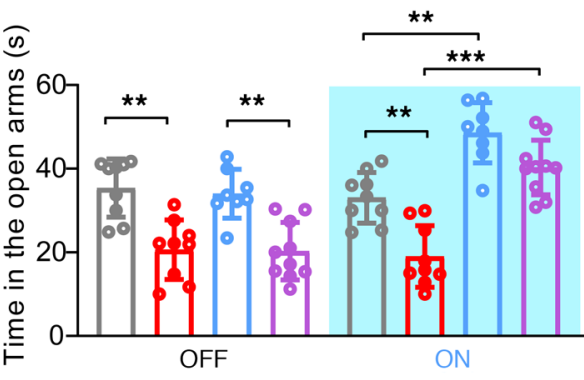

C

- Con-eYFP • Stress-eYFP

- Con-ChR2

- Stress-ChR2

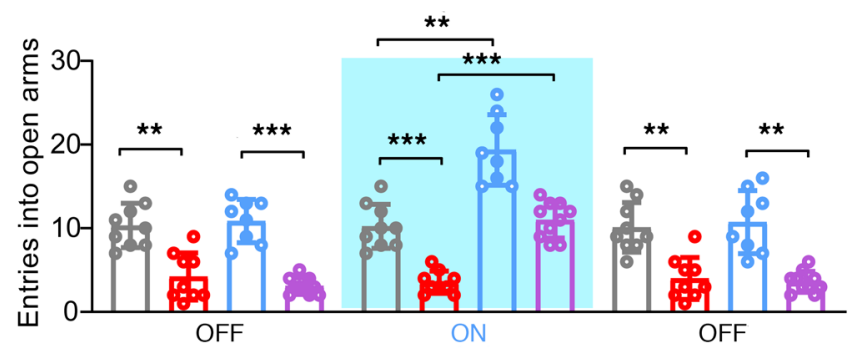

D
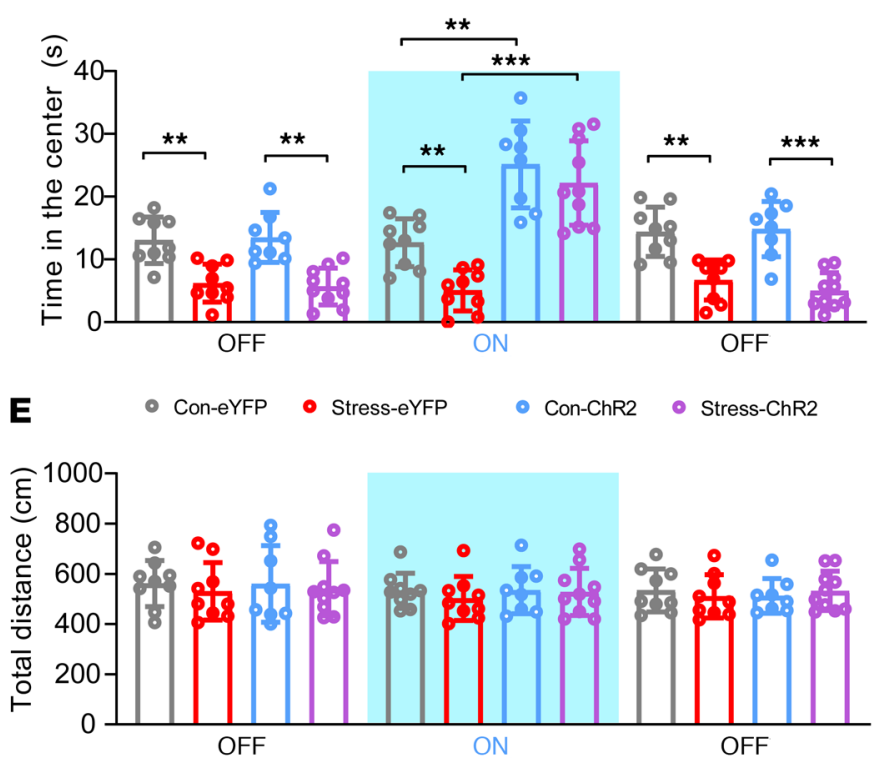

between the CeL and CeM in the control of fear extinction needs to be explored further.

With respect to the anxiety circuit as a whole, given the evolutionarily adaptive purposes of fear and anxiety for survival, it

Figure 8. IL-CeA projections reverse the anxiogenic effects of restraint stress. (A) Experimental paradigm. Neurons in the IL were transduced with either ChR2 or eYFP. Blue light was delivered via optical fibers implanted into the CeA after 3 days of restraint stress. (B-E) Compared with control mice, mice that underwent restraint stress showed a reduction of both open-arm exploration in the EPM test [B, $F_{\text {interaction }}$ $(6,63)=8.89, P<0.0001] ;\left[C, F_{\text {interaction }}(6,64)=11.40, P<\right.$ $0.0001]$ and center exploration in the OFT [D, $F$ $(6,64)=16.20, P<0.0001]$. IL-CeA activation reversed these stress-induced effects. No detectable effect was seen on the distance traveled by mice in each group $[\mathbf{E}$ $\left.F_{\text {interaction }}(6,64)=0.1177, P=0.9940\right] . n=9$ control-eYFP mice; $n=9$ stress-eYFP mice; $n=9$ control-ChR2 mice; $n=8$ stress-ChR2 mice. ${ }^{*} P<0.05,{ }^{*} P<0.01$, and ${ }^{* *} P<0.001$, by 2 -way, repeated-measures ANOVA with Tukey's multiple-comparison test (B-E). Data are presented as the mean \pm SEM. See Supplemental Table 1 for statistical details. Con, control.

is likely that anxiety circuits are widely distributed and highly redundant. This may explain why there are a host of parallel circuits in the brain that can contribute to the modulation of anxiety states. In the course of providing insight into fear and anxiety processes, this study identifies IL-LS and IL-CeA projections that represent forces oppositional to existing circuits in mediating anxiety-related behaviors. Other circuits to explore in the characterization of critical neural circuit elements of anxiety-related behaviors include the connections of the DP, the prelimbic cortex, the insular cortex, and projections from neuromodulatory regions.

\section{Methods}

We used commercially available C57BL/6J mice (obtained from the Guangdong Medical Laboratory Animal Center, Guangdong, China).

The procedures used for virus construction and packaging, virus injection, optical fiber and guide cannula placement, retrograde labeling, electrophysiological recordings, light delivery, drug delivery, behavioral assays, histology, and imaging are detailed in the Supplemental Methods.

Statistics. No specific method was used to predetermine the ideal sample size or to randomly assign the animals to the experimental groups. The sample sizes are indicated in Supplemental Table 1 and are similar to those reported in previous studies $(13,81)$. The normality of the data distribution was confirmed by the Shapiro-Wilk normality test. Statistical differences of normally distributed data were then determined using 2-way, repeated-measures ANOVA, Kruskal-Wallis ANOVA, or 1-way ANOVA followed by Bonferroni's post hoc analysis, 2-sided Mann-Whitney $U$ test, or Tukey's multiplecomparison test, as indicated in Supplemental Table 1. A P value of less 
than 0.05 was considered statistically significant. All data were analyzed with SPSS software (IBM).

\section{Study approval}

Ethics approval for this study was obtained from the ethics board for animal research of Southern Medical University (Guangzhou, China). The animals were handled in accordance with the Chinese Council on Animal Care Guidelines. Efforts were made to minimize animal suffering and to reduce the number of animals used.

\section{Author contributions}

TMG conceived the idea for this study, and TMG and YHC designed the study. YHC performed the experiments with the help of JLW, NYH, JPZ, SRZ, and WPL. JLW analyzed the electrophysiological data, and YHC analyzed all other data and generated the figures. XWL provided critical consumables. TMG and YHC interpreted the results with critical input from JMY, YHC, and TMG, and JMY wrote the manuscript.

\section{Acknowledgments}

This work was supported by grants from the National Natural Science Foundation of China (82090032 and 31830033, to TMG; 31801007 and 81881240049, to YHC); the Program for Changjiang Scholars and the Innovative Research Team in University (IRT_16R37, to TMG); the Key Area Research and Development Program of Guangdong Province (2018B030334001 and 2018B030340001, to TMG); the Guangdong Basic and Applied Basic Research Foundation(2020A1515011310, to YHC); the Guangdong-Hong Kong-Macao Greater Bay Area Center for Brain Science and Brain-Inspired Intelligence Fund (2019019, to YHC); and the Science and Technology Program of Guangzhou (202007030013, to TMG).

Address correspondence to: Tian-Ming Gao, Department of Neurobiology, Southern Medical University, 1023S Shatai Road, Guangzhou 510515, China. Phone: 86.20.61648617; Email: tgao@ smu.edu.cn.
1. Ressler KJ, Mayberg HS. Targeting abnormal neural circuits in mood and anxiety disorders: from the laboratory to the clinic. Nat Neurosci. 2007;10(9):1116-1124.

2. Kessler RC, et al. Lifetime prevalence and ageof-onset distributions of DSM-IV disorders in the National Comorbidity Survey Replication. Arch Gen Psychiatry. 2005;62(6):593-602.

3. Bluett EJ, et al. Acceptance and commitment therapy for anxiety and OCD spectrum disorders: an empirical review. J Anxiety Disord. 2014;28(6):612-624.

4. Clark DA, Beck AT. Cognitive theory and therapy of anxiety and depression: convergence with neurobiological findings. Trends Cogn Sci. 2010;14(9):418-424.

5. Warwick H, et al. Complete recovery from anxiety disorders following Cognitive Behavior Therapy in children and adolescents: a meta-analysis. Clin Psychol Rev. 2017;52:77-91.

6. Boggio PS, et al. Noninvasive brain stimulation with high-frequency and low-intensity repetitive transcranial magnetic stimulation treatment for posttraumatic stress disorder. JClin Psychiatry. 2010;71(8):992-999.

7. Osuch EA, et al. Repetitive TMS combined with exposure therapy for PTSD: a preliminary study. J Anxiety Disord. 2009;23(1):54-59.

8. Raij T, et al. Prefrontal cortex stimulation enhances fear extinction memory in humans. Biol Psychiatry. 2018;84(2):129-137.

9. DeNardo LA, et al. Temporal evolution of cortical ensembles promoting remote memory retrieval. Nat Neurosci. 2019;22(3):460-469.

10. Etkin A, et al. Emotional processing in anterior cingulate and medial prefrontal cortex. Trends Cogn Sci. 2011;15(2):85-93.

11. Fredrikson M, Faria V. Neuroimaging in anxiety disorders. Mod Trends Pharmacopsychiatry. 2013;29:47-66.

12. Sangha S, et al. Know safety, no fear. Neurosci Biobehav Rev. 2020;108:218-230.

13. Adhikari A, et al. Basomedial amygdala mediates top-down control of anxiety and fear. Nature. 2015;527(7577):179-185.
14. Bukalo O, et al. Prefrontal inputs to the amygdala instruct fear extinction memory formation. $\mathrm{Sci}$ Adv. 2015;1(6):e1500251.

15. Delgado MR, et al. Viewpoints: dialogues on the functional role of the ventromedial prefrontal cortex. Nat Neurosci. 2016;19(12):1545-1552.

16. Dejean C, et al. Neuronal circuits for fear expression and recovery: recent advances and potential therapeutic strategies. Biol Psychiatry. 2015;78(5):298-306.

17. Do-Monte FH, et al. A temporal shift in the circuits mediating retrieval of fear memory. Nature. 2015;519(7544):460-463.

18. Cho JH, et al. Synaptic encoding of fear extinction in $\mathrm{mPFC}$-amygdala circuits. Neuron. 2013;80(6):1491-1507.

19. Corcoran KA, Quirk GJ. Activity in prelimbic cortex is necessary for the expression of learned, but not innate, fears. J Neurosci. 2007;27(4):840-844.

20. Sierra-Mercado D, et al. Inactivation of the ventromedial prefrontal cortex reduces expression of conditioned fear and impairs subsequent recall of extinction. Eur J Neurosci. 2006;24(6):1751-1758.

21. Vidal-Gonzalez I, et al. Microstimulation reveals opposing influences of prelimbic and infralimbic cortex on the expression of conditioned fear. Learn Mem. 2006;13(6):728-733.

22. Ji G, Neugebauer V. Modulation of medial prefrontal cortical activity using in vivo recordings and optogenetics. Mol Brain. 2012;5:36.

23. Lacroix L, et al. Differential role of the medial and lateral prefrontal cortices in fear and anxiety. Behav Neurosci. 2000;114(6):1119-1130.

24. Sullivan RM, Gratton A. Behavioral effects of excitotoxic lesions of ventral medial prefrontal cortex in the rat are hemisphere-dependent. Brain Res. 2002;927(1):69-79.

25. Bi LL, et al. Enhanced excitability in the infralimbic cortex produces anxiety-like behaviors. Neuropharmacology. 2013;72:148-156.

26. Milad MR, et al. Electrical stimulation of medial prefrontal cortex reduces conditioned fear in a temporally specific manner. Behav Neurosci. 2004;118(2):389-394
27. Laurent V, Westbrook RF. Inactivation of the infralimbic but not the prelimbic cortex impairs consolidation and retrieval of fear extinction. Learn Mem. 2009;16(9):520-529.

28. Vertes RP. Differential projections of the infralimbic and prelimbic cortex in the rat. Synapse. 2004;51(1):32-58.

29. Wood M, et al. Infralimbic prefrontal cortex structural and functional connectivity with the limbic forebrain: a combined viral genetic and optogenetic analysis. Brain Struct Funct. 2019;224(1):73-97.

30. Fenno L, et al. The development and application of optogenetics. Annu Rev Neurosci. 2011;34:389-412.

31. Do-Monte FH, et al. Revisiting the role of infralimbic cortex in fear extinction with optogenetics. J Neurosci. 2015;35(8):3607-3615.

32. Tovote $P$, et al. Neuronal circuits for fear and anxiety. Nat Rev Neurosci. 2015;16(6):317-331.

33. Calhoon GG, Tye KM. Resolving the neural circuits of anxiety. Nat Neurosci. 2015;18(10):1394-1404.

34. Anthony TE, et al. Control of stress-induced persistent anxiety by an extra-amygdala septohypothalamic circuit. Cell. 2014;156(3):522-536.

35. Tye KM, et al. Amygdala circuitry mediating reversible and bidirectional control of anxiety. Nature. 2011;471(7338):358-362.

36. Ciocchi S, et al. Encoding of conditioned fear in central amygdala inhibitory circuits. Nature. 2010;468(7321):277-282.

37. Haubensak W, et al. Genetic dissection of an amygdala microcircuit that gates conditioned fear. Nature. 2010;468(7321):270-276.

38. Li H, et al. Experience-dependent modification of a central amygdala fear circuit. Nat Neurosci. 2013;16(3):332-339.

39. Burger C, et al. Recombinant AAV viral vectors pseudotyped with viral capsids from serotypes 1,2 , and 5 display differential efficiency and cell tropism after delivery to different regions of the central nervous system. Mol Ther. 2004;10(2):302-317.

40. Aschauer DF, et al. Analysis of transduction 
efficiency, tropism and axonal transport of AAV serotypes 1, 2, 5, 6, 8 and 9 in the mouse brain. PLoS One. 2013;8(9):e76310.

41. Yizhar O, et al. Optogenetics in neural systems. Neuron. 2011;71(1):9-34.

42. Carola V, et al. 2002. Evaluation of the elevated plus-maze and open-field tests for the assessment of anxiety-related behavior in inbred mice. Behav Brain Res. 2002;134(1-2):49-57.

43. Kim SY, et al. Diverging neural pathways assemble a behavioral state from separable features in anxiety. Nature. 2013;496(7444):219-223.

44. Lerner TN, et al. Intact-brain analyses reveal distinct information carried by SNc dopamine subcircuits. Cell. 2015;162(3):635-647.

45. Soudais C, et al. Preferential transduction of neurons by canine adenovirus vectors and their efficient retrograde transport in vivo. FASEB J. 2001;15(12):2283-2285.

46. Mahn M, et al. Biophysical constraints of optogenetic inhibition at presynaptic terminals. Nat Neurosci. 2016;19(4):554-556.

47. Armbruster BN, et al. Evolving the lock to fit the key to create a family of $G$ protein-coupled receptors potently activated by an inert ligand. Proc Natl Acad Sci U S A. 2007;104(12):5163-5168.

48. Peters J, et al. Induction of fear extinction with hippocampal-infralimbic BDNF. Science. 2010;328(5983):1288-1290.

49. Mueller D, et al. Infralimbic D2 receptors are necessary for fear extinction and extinction-related tone responses. Biol Psychiatry. 2010;68(11):1055-1060.

50. Quirk GJ, Mueller D. Neural mechanisms of extinction learning and retrieval. Neuropsychopharmacology. 2008;33(1):56-72.

51. Milad MR, Quirk GJ. Neurons in medial prefrontal cortex signal memory for fear extinction. Nature. 2002;420(6911):70-74.

52. Soria-Gomez E, et al. Habenular CB1 receptors control the expression of aversive memories. Neuron. 2015;88(2):306-313.

53. Cassell MD, et al. The intrinsic organization of the central extended amygdala. Ann N Y Acad Sci. 1999;877:217-241.

54. Rosenkranz JA, Grace AA. Dopamine attenuates prefrontal cortical suppression of sensory inputs to the basolateral amygdala of rats. JNeurosci.
2001;21(11):4090-4103.

55. Motzkin JC, et al. Ventromedial prefrontal cortex is critical for the regulation of amygdala activity in humans. Biol Psychiatry. 2015;77(3):276-284.

56. Quirk GJ, et al. Stimulation of medial prefrontal cortex decreases the responsiveness of central amygdala output neurons. J Neurosci. 2003;23(25):8800-8807.

57. Rosenkranz JA, et al. The prefrontal cortex regulates lateral amygdala neuronal plasticity and responses to previously conditioned stimuli. J Neurosci. 2003;23(35):11054-11064.

58. Adhikari A, et al. Single units in the medial prefrontal cortex with anxiety-related firing patterns are preferentially influenced by ventral hippocampal activity. Neuron. 2011;71(5):898-910.

59. Adhikari A, et al. Synchronized activity between the ventral hippocampus and the medial prefrontal cortex during anxiety. Neuron 2010;65(2):257-269.

60. Jennings JH, et al. Distinct extended amygdala circuits for divergent motivational states. Nature. 2013;496(7444):224-228.

61. Senn V, et al. Long-range connectivity defines behavioral specificity of amygdala neurons. Neuron. 2014;81(2):428-437.

62. Ahrlund-Richter S, et al. A whole-brain atlas of monosynaptic input targeting four different cell types in the medial prefrontal cortex of the mouse. Nat Neurosci. 2019;22(4):657-668.

63. Sun $\mathrm{Q}$, et al. A whole-brain map of long-range inputs to GABAergic interneurons in the mouse medial prefrontal cortex. Nat Neurosci. 2019;22(8):1357-1370.

64. Hoover WB, Vertes RP. Anatomical analysis of afferent projections to the medial prefrontal cortex in the rat. Brain Struct Funct. 2007;212(2):149-179.

65. Zucker RS, Regehr WG. Short-term synaptic plasticity. Annu Rev Physiol. 64:355-405.

66. Griffith WH. Voltage-clamp analysis of posttetanic potentiation of the mossy fiber to CA3 synapse in hippocampus. J Neurophysiol. 1990;63(3):491-501.

67. Sheehan TP, et al. Regulation of affect by the lateral septum: implications for neuropsychiatry. Brain Res Brain Res Rev. 2004;46(1):71-117.

68. Degroot A, et al. Septal GABAergic and hippocampal cholinergic systems modulate anxiety in the plus-maze and shock-probe tests. Pharmacol Biochem Behav. 2001;69(3-4):391-399.

69. Albert DJ, Walsh ML. The inhibitory modulation of agonistic behavior in the rat brain: a review. Neurosci Biobehav Rev. 1982;6(2):125-143.

70. Menard J, Treit D. Effects of centrally administered anxiolytic compounds in animal models of anxiety. Neurosci Biobehav Rev. 1999;23(4):591-613.

71. Pesold C, Treit D. The neuroanatomical specificity of the anxiolytic effects of intra-septal infusions of midazolam. Brain Res. 1996;710(12):161-168

72. Risold PY, Swanson LW. Chemoarchitecture of the rat lateral septal nucleus. Brain Res Brain Res Rev. 1997;24(2-3):91-113.

73. Parfitt GM, et al. Bidirectional control of anxiety-related behaviors in mice: role of inputs arising from the ventral hippocampus to the lateral septum and medial prefrontal cortex. Neuropsychopharmacology. 2017;42(8):1715-1728.

74. Yadin E, et al. The role of the lateral septum in anxiolysis. Physiol Behav. 1993;53(6):1077-1083.

75. Kalin NH, et al. The role of the central nucleus of the amygdala in mediating fear and anxiety in the primate. J Neurosci. 2004;24(24):5506-5515.

76. Etkin A, et al. Disrupted amygdalar subregion functional connectivity and evidence of a compensatory network in generalized anxiety disorder. Arch Gen Psychiatry. 2009;66(12):1361-1372.

77. Fu JY, et al. Whole-brain map of long-range monosynaptic inputs to different cell types in the amygdala of the mouse. Neurosci Bull. 2020;36(11):1381-1394.

78. Pitkanen A, et al. Organization of intra-amygdaloid circuitries in the rat: an emerging framework for understanding functions of the amygdala. Trends Neurosci. 1997;20(11):517-523.

79. Cassell MD, et al. Neuronal architecture in the rat central nucleus of the amygdala: a cytological, hodological, and immunocytochemical study. J Comp Neurol. 1986;246(4):478-499.

80. Huber D, et al. Vasopressin and oxytocin excite distinct neuronal populations in the central amygdala. Science. 2005;308(5719):245-248.

81. Felix-Ortiz AC, et al. BLA to vHPC inputs modulate anxiety-related behaviors. Neuron. 2013;79(4):658-664. 